\title{
A Structural Model for Binding of the Serine-Rich Repeat Adhesin GspB to Host Carbohydrate Receptors
}

\author{
Tasia M. Pyburn ${ }^{1,2}$, Barbara A. Bensing ${ }^{3}$, Yan Q. Xiong ${ }^{4}$, Bruce J. Melancon ${ }^{2,5 \times a}$, Thomas M. \\ Tomasiak $^{1,2 \times b}$, Nicholas J. Ward'1, Victoria Yankovskaya ${ }^{6}$, Kevin M. Oliver ${ }^{2,5}$, Gary Cecchini ${ }^{6,7}$, Gary A. \\ Sulikowski $^{2,5}$, Matthew J. Tyska ${ }^{8}$, Paul M. Sullam ${ }^{3}$, T. M. Iverson ${ }^{1,2,9 *}$
}

\begin{abstract}
1 Department of Pharmacology, Vanderbilt University Medical Center, Nashville, Tennessee, United States of America, 2 Vanderbilt Institute of Chemical Biology, Nashville, Tennessee, United States of America, 3 Department of Medicine, Veterans Affairs Medical Center and the University of California, San Francisco, California, United States of America, 4 Department of Medicine, Harbor-UCLA Medical Center, Torrance, California, United States of America, 5 Department of Chemistry, Vanderbilt University, Nashville, Tennessee, United States of America, 6 Molecular Biology Division, Veterans Affairs Medical Center, San Francisco, California, United States of America, 7 Department of Biochemistry \& Biophysics University of California, San Francisco, California, United States of America, 8 Department of Cell and Developmental Biology, Vanderbilt University Medical Center, Nashville, Tennessee, United States of America, 9 Department of Biochemistry, Vanderbilt University Medical Center, Nashville, Tennessee, United States of America
\end{abstract}

\begin{abstract}
GspB is a serine-rich repeat (SRR) adhesin of Streptococcus gordonii that mediates binding of this organism to human platelets via its interaction with sialyl-T antigen on the receptor GPIb $\alpha$. This interaction appears to be a major virulence determinant in the pathogenesis of infective endocarditis. To address the mechanism by which GspB recognizes its carbohydrate ligand, we determined the high-resolution $\mathrm{x}$-ray crystal structure of the $\mathrm{GspB}$ binding region $\left(\mathrm{GspB} \mathrm{BR}_{\mathrm{BR}}\right.$, both alone and in complex with a disaccharide precursor to sialyl-T antigen. Analysis of the GspB $B_{B R}$ structure revealed that it is comprised of three independently folded subdomains or modules: 1) an Ig-fold resembling a CnaA domain from prokaryotic pathogens; 2) a second Ig-fold resembling the binding region of mammalian Siglecs; 3) a subdomain of unique fold. The disaccharide was found to bind in a pocket within the Siglec subdomain, but at a site distinct from that observed in mammalian Siglecs. Confirming the biological relevance of this binding pocket, we produced three isogenic variants of $S$. gordonii, each containing a single point mutation of a residue lining this binding pocket. These variants have reduced binding to carbohydrates of GPlb $\alpha$. Further examination of purified $\mathrm{GspB}_{\mathrm{BR}}-\mathrm{R} 484 \mathrm{E}$ showed reduced binding to sialyl-T antigen while $S$. gordonii harboring this mutation did not efficiently bind platelets and showed a significant reduction in virulence, as measured by an animal model of endocarditis. Analysis of other SRR proteins revealed that the predicted binding regions of these adhesins also had a modular organization, with those known to bind carbohydrate receptors having modules homologous to the Siglec and Unique subdomains of $\mathrm{GspB}_{\mathrm{BR}}$. This suggests that the binding specificity of the SRR family of adhesins is determined by the type and organization of discrete modules within the binding domains, which may affect the tropism of organisms for different tissues.
\end{abstract}

Citation: Pyburn TM, Bensing BA, Xiong YQ, Melancon BJ, Tomasiak TM, et al. (2011) A Structural Model for Binding of the Serine-Rich Repeat Adhesin GspB to Host Carbohydrate Receptors. PLoS Pathog 7(7): e1002112. doi:10.1371/journal.ppat.1002112

Editor: Partho Ghosh, University of California San Diego, United States of America

Received January 17, 2011; Accepted April 25, 2011; Published July 7, 2011

Copyright: (c) 2011 Pyburn et al. This is an open-access article distributed under the terms of the Creative Commons Attribution License, which permits unrestricted use, distribution, and reproduction in any medium, provided the original author and source are credited.

Funding: This work was supported by the American Heart Association grants 09GRNT2180065 (YQX), 09GRNT2310188 (MJT), and 09GRNT2220122 (TMI), Pilot Project funds from the Vanderbilt Institute of Chemical Biology (TMI and GAS), Pilot funds from the VICTR CTSA UL1 RR024975 from NCRR/NIH (TMI), Vanderbilt University IDEAs award (MJT), the Department of Veterans Affairs (PMS and GC), and grants GM079419 (TMI), AI079558 (TMI), AI041513 (PMS), AI057433 (PMS), DK075555 (MJT) and GM61606 (GC) from the National Institutes of Health. TMT and KMO were supported by T32 GM65086 (Chemical-Biology Interface Training Grant). BJM was supported by T90 DA022873 (Integrative Training in Therapeutic Discovery Training Grant). Portions of this research were carried out at beamlines 9-2, 11-1, and 12-2 at Stanford Synchrotron Radiation Lightsource (SSRL), a national user facility operated by Stanford University on behalf of the U.S. Department of Energy, Office of Basic Energy Sciences. The SSRL Structural Molecular Biology Program is supported by the Department of Energy, Office of Biological and Environmental Research, and by the National Institutes of Health, National Center for Research Resources, Biomedical Technology Program, and the National Institute of General Medical Sciences. The Life Sciences Collaborative Access Team (LS-CAT) ID21-D, ID21-F, ID21-G at Advanced Photon Source (APS). Use of the Advanced Photon Source was supported by the U. S. Department of Energy, Office of Science, Office of Basic Energy Sciences, under Contract No. DE-AC0206CH11357. Use of the LS-CAT Sector 21 was supported by the Michigan Economic Development Corporation and the Michigan Technology Tri-Corridor for the support of this research program (Grant 085P1000817).

Competing Interests: The authors have declared that no competing interests exist.

*E-mail: tina.iverson@vanderbilt.edu

aa Current address: Vanderbilt Program in Drug Discovery, Department of Pharmacology, Nashville, Tennessee, United States of America

ab Current address: Molecular Structure Group, University of California, San Francisco, California, United States of America

\section{Introduction}

The serine-rich repeat (SRR) glycoproteins of Gram-positive bacteria are an expanding family of microbial adhesins and virulence factors [1-6]. These proteins consist of a distinctive signal sequence and export-targeting region at the N-terminus, a short SRR region ( $\sim 50-170$ amino acids), a ligand binding region, a second lengthy SRR region ( $\sim 400-4000$ amino acids), and a cell wall anchoring motif at the C-terminus (Fig. 1) [1,2,4,6-9]. The binding regions of the SRR glycoproteins contain significant sequence variation, which appears to account for their broad range of binding targets, including platelet membrane and salivary 


\section{Author Summary}

The binding of bacteria to human platelets is thought to be important for development of infective endocarditis, a life-threatening infection of the cardiovascular system. Streptococcus gordonii is a leading cause of endocarditis. This pathogen uses a protein called GspB to attach to carbohydrates on human platelets. While this binding interaction appears to be mediated by a specific, contiguous domain within GspB, little is known about the molecular details of the interaction between GspB and the carbohydrate receptors on its human host. We therefore determined the crystal structure of the region of GspB that binds to platelet carbohydrates, both alone and in complex with a synthetic carbohydrate receptor. Using this structure as a guide, we were able to produce three strains of S. gordonii that lacked the ability to bind to platelet carbohydrates. One of these isogenic variants was studied more in-depth and lacked the ability to bind to human platelets in vitro and was reduced in virulence when tested in vivo. These studies provide the first structural information detailing the molecular interactions between any serine-rich repeat adhesin and its host receptor, and identify how different, related adhesins may have evolved different specificities for host receptors.

glycoproteins [1,4,9-11], endothelial cells [12], epithelial cells [13], erythrocytes [14,15], and keratins [16,17]. Expression of SRR proteins has been associated with increased virulence in several animal models of infection, including endocarditis $[6,18]$, meningitis [12], pneumonia [5], and bacteremia [7,19].

A number of bacterial surface components have been shown to mediate platelet binding in vitro, either by interacting directly with receptors on the platelet membrane, or via bridging molecules, such as fibrinogen [20-29]. The contribution of these interactions to virulence, however, has been assessed for relatively few of these adhesins. Previous studies have focused on the molecular basis for the SRR adhesin mediated binding of gram-positive bacteria to human platelets, and the role of this process in the pathogenesis of infective endocarditis. This interaction appears to be important for the attachment of blood-borne bacteria to platelets on the surface of damaged cardiac valves, thereby initiating infection. In addition, the subsequent deposition of platelets onto the infected endocardium may be due in part to bacterium-platelet binding, resulting in the formation macroscopic vegetations, which are the hallmark lesions of this disease [30]. Three of the SRR proteins (GspB of Streptococcus gordonii strain M99, Hsa of S. gordonii strain Challis, and SrpA of Streptococcus sanguinis strain SK36) bind human platelets through their interaction with glycocalicin, which is the carbohydrate-rich extracellular portion of the platelet membrane glycoprotein GPIb $\alpha$ [9,11]. While the specific carbohydrate receptor for SrpA has not yet been identified, GspB and Hsa recognize sialylated trisaccharides $[1,11,31,32]$. Dot blot assays using immobilized carbohydrates have demonstrated that GspB has high fidelity for sialyl-T antigen (i.e. NeuAc $\alpha(2-3) \mathrm{Gal} \beta(1-$ 3)GalNAc), one of the major carbohydrates on GPIb $\alpha$ [11], while Hsa binds to glycocalicin via either sialyl-T antigen or sialyllactose (Neu5Ac $\alpha(2-6) \operatorname{Gal} \beta(1-4)$ Glc) $[31,32]$. Binding of these SRR adhesins to platelets is a high affinity process, with the interaction between $\mathrm{GspB}$ and glycocalicin having a $\mathrm{K}_{\mathrm{D}}$ of $2.38 \times 10^{-8} \mathrm{M}$ [11] and appears to be a major factor in the pathogenesis of infective endocarditis, since the loss of GspB or Hsa expression results in a marked reduction in virulence [33].

Structural information can enhance the understanding of the determinants of binding specificity. Here, we report the highresolution crystal structure of $\mathrm{GspB}_{\mathrm{BR}}$, both alone and in complex with the disaccharide $\alpha$-2,3-sialyl (1-thioethyl)galactose, a precursor to synthetically-produced sialyl-T antigen. From these structures, we identified that a subdomain of $\mathrm{GspB}_{\mathrm{BR}}$ resembling mammalian sialic acid binding proteins binds to $\alpha$-2,3-sialyl (1thioethyl)galactose. Site directed mutagenesis and in vivo studies in a rat model of infective endocarditis verified that this carbohydrate binding site within $\mathrm{GspB}_{\mathrm{BR}}$ mediates binding of $S$. gordonii strain M99 to sialyl-T antigen, the host carbohydrates of the platelet

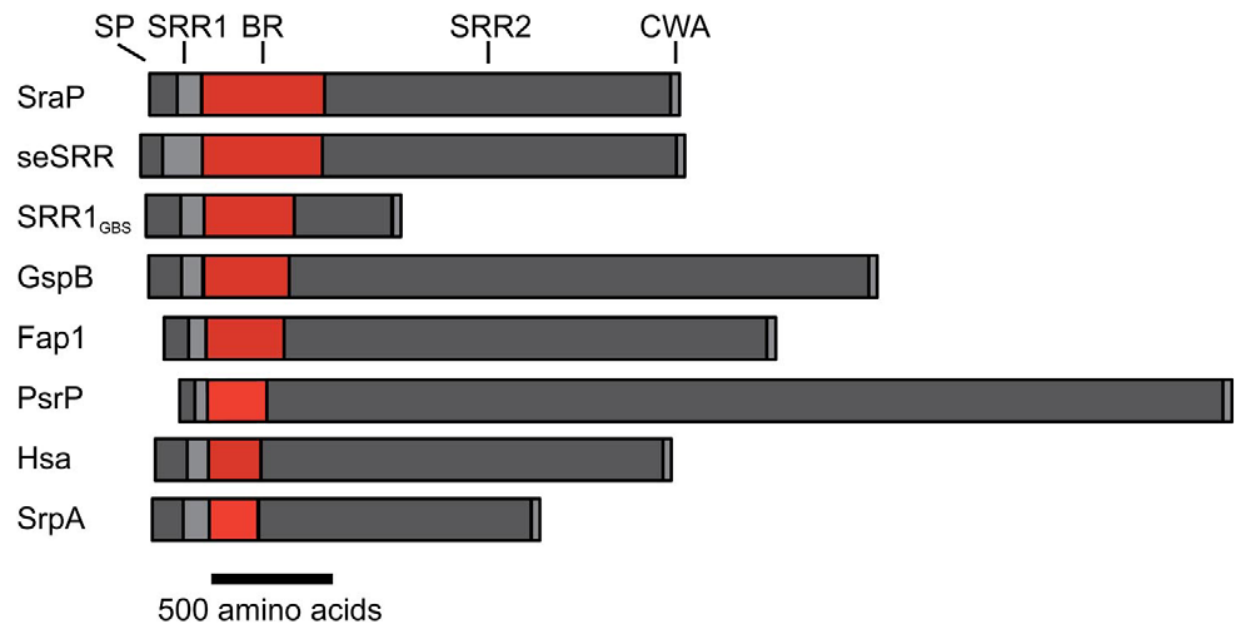

Figure 1. Schematic of the SRR adhesins. Selected SRR adhesins are aligned based on the N-terminus of their binding regions, with the largest binding region at the top and the smallest binding region at the bottom. The length of each domain and the entire protein is drawn to-scale. $\mathrm{SP}=$ signal peptide (dark gray), SRR1 = first serine rich repeat (light gray), BR=binding region (red), SRR2 = second serine rich repeat (dark gray), CWA = cell wall anchoring motif (light gray). The SRR adhesins used in this figure are S. aureus SraP (SraP); S. epidermidis SRR1 (seSRR), S. agalactiae SRR1 (SRR1 $1_{G B S}$ ), S. gordonii strain M99 GspB (GspB), S. parasanguinis Fap1 (Fap1), S. pneumoniae PsrP (PsrP), S. gordonii strain Challis Hsa (Hsa), and S. sanguinis SrpA (SrpA).

doi:10.1371/journal.ppat.1002112.g001 
membrane receptor GPIb $\alpha$, and intact platelets, and that this interaction is important for virulence. Our analysis of the structure further identified that $\mathrm{GspB}_{\mathrm{BR}}$ contains an unusually modular fold, prompting us to re-analyze the sequences of the SRR family of adhesins. We determined that other structurally uncharacterized SRR adhesins also contain modules within their binding regions, suggesting that particular subdomains may be included, removed, or interchanged, manifesting in the broad range of binding partners observed in the family.

\section{Results}

\section{Overall structure of $\mathrm{GspB}_{\mathrm{BR}}$}

We determined the structure of $\mathrm{GspB}_{\mathrm{BR}}$ to $1.4 \AA$ resolution using the method of Multiwavelength Anomalous Dispersion from a single $\mathrm{Dy}^{3+}$ derivative (Fig. 2, Table 1, Table 2). $\mathrm{GspB}_{\mathrm{BR}}$ folds into an elongated rod, with dimensions of $\sim 130 \AA \times 30 \AA \times 30 \AA$. This rod is comprised of three apparently independently-folded subdomains arranged in a linear fashion, like beads on a string. The secondary structure of each of the three subdomains is predominated by $\beta$-strands. Interestingly, the first two subdomains are organized around core folds that resemble those found within the eukaryotic immunoglobulin $(\mathrm{Ig})$ superfamily $(\mathbf{F i g}$. 3). Ig-folds have previously been identified in prokaryotic proteins [34-41], and it has been noted that some of these bacterial proteins with Igfolding topologies contain sequence similarity to their eukaryotic counterparts, while the others lack the residues conserved in the core of eukaryotic proteins with Ig-folds. $\mathrm{GspB}_{\mathrm{BR}}$ has homology with bacterial proteins that do not contain detectable sequence similarity to eukaryotic proteins with Ig-folds, lacking even the cysteines that normally form a disulfide bond.

Several topology variants of Ig-folds have been characterized. A structural homology search using the EMBL DaliLite server [42] identified that the N-terminal subdomain of $\mathrm{GspB}_{\mathrm{BR}}$ contains a folding topology, strand inserts, and inter-sheet angle reminiscent of the DE-variant of the Ig-fold [36] (Fig. 3A-G). This Ig-fold topology is found in prokaryotic proteins and was first identified within the Aregion of the Staphylococcus aureus GNA protein [35]. CNA belongs to the family of microbial surface components recognizing adhesive matrix molecules (MSCRAMMs) of Gram-positive pathogens [3436]. Given its structural similarity, this N-terminal subdomain of $\mathrm{GspB}_{\mathrm{BR}}$ will be termed the CnaA subdomain. The highest structural similarity between the CnaA subdomain of $\mathrm{GspB}_{\mathrm{BR}}$ and any other structurally characterized protein is to the C-terminal subdomain of the binding region of SRR adhesin Fapl from Streptococcus parasanguinis $\left(\mathrm{Fapl}_{\mathrm{NR}}-\boldsymbol{\beta}\right)$ [43]. The RMS deviation of the structural alignment between the $\mathrm{CnaA}$ subdomain of $\mathrm{GspB}_{\mathrm{BR}}$ and the Fapl $_{\mathrm{NR}}-\beta$ subdomain is $2.5 \AA$ for $200 \mathrm{C}_{\alpha}$ atoms.
A

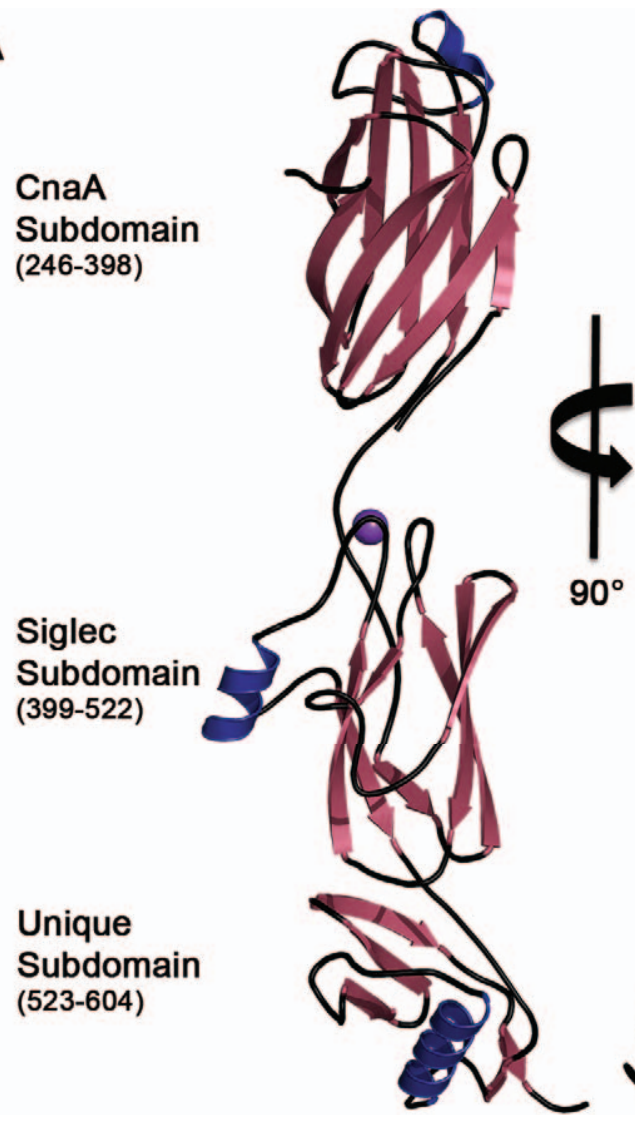

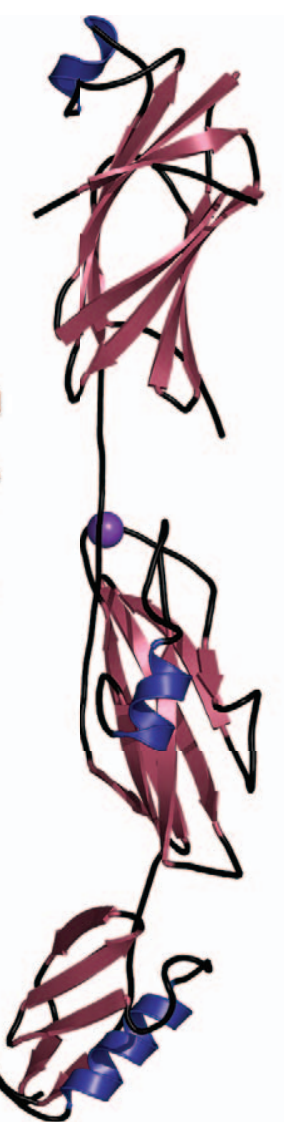

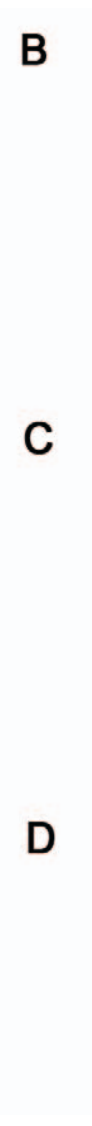

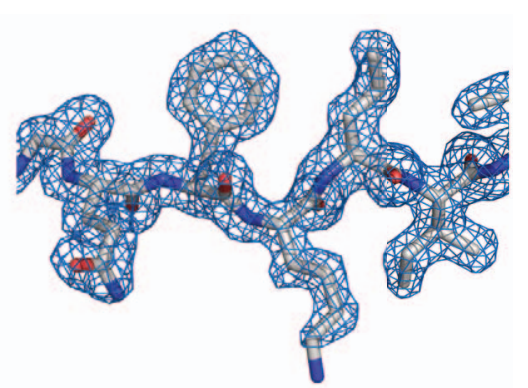
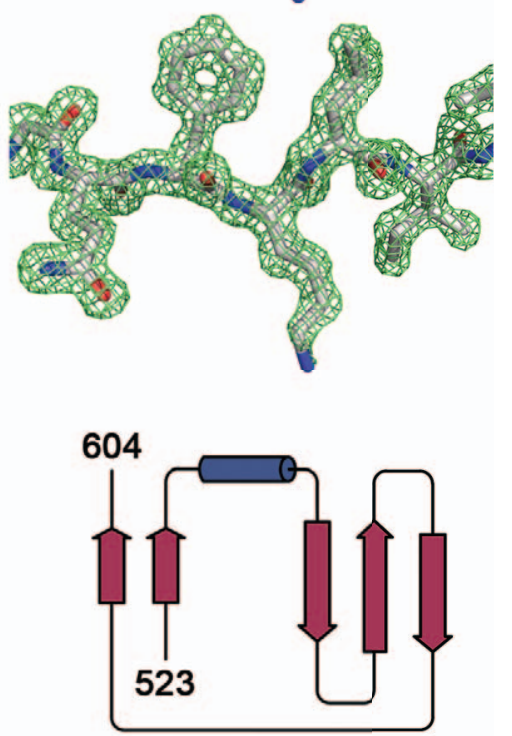

Figure 2. Structure of $\mathbf{G s p B}_{\mathbf{B R}}$. A. Two views of the structure of $\mathrm{GspB}_{\mathrm{BR}}$ separated by a $90^{\circ}$ rotation. The $\mathrm{N}$-terminus of the binding region is located at the top of the figure and the C-terminus is located at the bottom. $\beta$-Strands are colored magenta, $\alpha$-helices are colored blue, and loops are colored black. A cation bound within the Siglec domain is shown as a purple sphere. B. Representative experimental electron density (blue mesh) at $2.0 \AA$ resolution, contoured at $1.0 \sigma$, and depicted superpositioned onto residues $515-520$ of the final model. C. A m $\left|\mathrm{F}_{\mathrm{o}}\right|-\mathrm{d}\left|\mathrm{F}_{\mathrm{c}}\right|$ omit electron density map at $1.4 \AA$ resolution (green mesh) calculated in REFMAC5 [65] after the removal of the model contoured at $3.0 \sigma$ and depicted superpositioned onto residues 515-520 of the final model. D. Folding diagram of the Unique subdomain. $\beta$-Strands are colored magenta and the $\alpha$-helix is colored blue.

doi:10.1371/journal.ppat.1002112.g002 
Table 1. X-ray data collection and refinement statistics.

\begin{tabular}{|c|c|c|c|}
\hline \multirow[t]{2}{*}{$\begin{array}{l}\text { PDB Accession } \\
\text { Number }\end{array}$} & \multirow{2}{*}{$\frac{3 Q C 5}{\text { Native }}$} & \multirow{2}{*}{$\frac{3 Q C 6}{\text { Native2 }}$} & \multirow{2}{*}{$\frac{\text { 3QD1 }}{\text { Disaccharide }}$} \\
\hline & & & \\
\hline Space group & $\mathrm{P} 2{ }_{1} 2_{1} 2_{1}$ & $\mathrm{P} 2{ }_{1} 2_{1} 2_{1}$ & $\mathrm{P} 2_{1}$ \\
\hline Wavelength (Å) & 0.97856 & 1.03034 & 1.07806 \\
\hline Beamline & LS-CAT ID-21-F & SSRL 11-1 & LS-CAT ID-21-D \\
\hline Resolution (Å) & $50-1.40$ & $50-1.90$ & $50-1.90$ \\
\hline Unit-cell a,b,c ( $(\AA)$ & $33.7,96.8,100.2$ & $33.3,86.7,117.9$ & $69.9,34.0,83.4$ \\
\hline $\begin{array}{l}\text { No. of measured } \\
\text { reflections }\end{array}$ & 223296 & 84235 & 97430 \\
\hline $\begin{array}{l}\text { No. of unique } \\
\text { reflections }\end{array}$ & 60816 & 26193 & 29986 \\
\hline Multiplicity & $3.7(2.0)^{1}$ & $3.2(2.9)$ & $3.2(2.6)$ \\
\hline$I_{\text {mean }} / \sigma(\mathrm{I})$ & 19.8(2.9) & 16.8(3.9) & $17.4(4.8)$ \\
\hline Completeness (\%) & $92.7(54.1)$ & 93.8(92.4) & 96.3(93.0) \\
\hline $\mathrm{R}_{\text {sym }}^{2}(\%)$ & $4.1(24.4)$ & $6.8(35.3)$ & 8.9(35.6) \\
\hline $\mathrm{R}_{\text {work }}{ }^{3} / \mathrm{R}_{\text {free }}{ }^{4}(\%)$ & $14.2 / 17.2$ & $21.5 / 26.1$ & $17.8 / 21.4$ \\
\hline $\begin{array}{l}\text { No. of reflections } \\
\text { in test set }\end{array}$ & 2543 & 1265 & 1477 \\
\hline No. of protein atoms & 352 & 351 & 359 \\
\hline No. of water atoms & 400 & 231 & 212 \\
\hline $\begin{array}{l}\text { RMS deviation bonds } \\
(\AA)\end{array}$ & 0.012 & 0.013 & 0.12 \\
\hline $\begin{array}{l}\text { RMS deviation angles } \\
\left({ }^{\circ}\right)\end{array}$ & 1.38 & 1.36 & 1.30 \\
\hline \multicolumn{4}{|c|}{$\begin{array}{l}{ }^{1} \text { Numbers in parentheses indicate values for the highest resolution bin. } \\
{ }^{2} R_{\text {sym }}=\sum\left[l_{i}-<I>\right](100) / \sum[<I>] \text { where } i \text { is ith measurement and }<I>\text { is the } \\
\text { weighted mean of } I . \\
{ }^{3} R_{\text {work }}=\sum \|\left|F_{\text {obs }}\right|-\left|F_{\text {calc }}\right|\left|\sum\right| F_{\text {obs }} \mid \\
{ }^{4} R_{\text {free }} \text { is calculated using the same equation as for } R_{\text {work }} \text { but using only the } \\
\text { reflections randomly omitted from refinement reserved in the test set of the } \\
\text { data. } \\
\text { doi:10.1371/journal.ppat.1002112.t001 }\end{array}$} \\
\hline
\end{tabular}

Surprisingly, a structural homology search using EMBL DaliLite [42] identified that the second subdomain contained a topology and strand inserts reminiscent of the $\mathrm{V}$-set $\mathrm{Ig}$ fold adopted by eukaryotic sialic acid binding immunoglobulin-like lectins [44-46] (Siglecs; Fig. 3D-F). However, the $\mathrm{C}^{\prime}$ and $\mathrm{C}^{\prime \prime}$ strands normally inserted into the $\mathrm{V}$-set Ig-fold are instead replaced by a long loop inserted at the same location (Fig. 3DF). The RMS deviation of the structural alignment between the second subdomain of $\mathrm{GspB}_{\mathrm{BR}}$ and Siglec-5 is $2.9 \AA$ for $210 \mathrm{C}_{\boldsymbol{\alpha}}$ atoms despite only $6 \%$ sequence identity. Like GspB, Siglecs bind carbohydrate receptors. Accordingly, the second domain is termed the Siglec subdomain. This subdomain of $\mathrm{GspB}_{\mathrm{BR}}$ contained electron density consistent with a cation-binding site (see Supporting Text S1, Fig. S1, Supporting Protocol S1). This was rather unexpected since Siglecs themselves do not bind cations in the carbohydrate-binding domain. The seven-coordinate number suggests that under physiological conditions, this should be a $\mathrm{Ca}^{2+}$ binding site. We explored the role of $\mathrm{Ca}^{2+}$ and other cations for the binding of $S$. gordonii strain M99 to glycocalicin. However, metal depletion, metal substitution, and site directed mutagenesis of the residues coordinating the ion were all consistent with the cation not being essential for carbohydrate receptor recognition (data not shown).

While still predominated by $\beta$-strands, the C-terminal subdomain of $\mathrm{GspB}_{\mathrm{BR}}$ does not contain an Ig-fold. In fact, a structural homology
Table 2. Derivative data collection and phasing statistics.

\begin{tabular}{|c|c|c|c|}
\hline & Peak & Inflection & Remote \\
\hline Space group & $\mathrm{P} 22_{1} 2_{1} 2_{1}$ & $P 2{ }_{1} 2_{1} 2_{1}$ & $P 2{ }_{1} 2_{1} 2_{1}$ \\
\hline Wavelength $(\AA)$ & 1.59083 & 1.59122 & 1.02463 \\
\hline Beamline & SSRL 9-2 & SSRL 9-2 & SSRL 9-2 \\
\hline Resolution $(\AA ̊)$ & 50-1.98 & $50-1.98$ & 50-1.55 \\
\hline Unit-cell a,b,c ( $(\AA)$ & $35.5,98.5,99.1$ & $34.5,98.5,99.1$ & $34.5,98.5,99.0$ \\
\hline $\begin{array}{l}\text { No. of measured } \\
\text { reflections }\end{array}$ & 203951 & 206064 & 431338 \\
\hline No. of unique reflections & 23918 & 23929 & 48691 \\
\hline Multiplicity & $8.5(8.1)^{1}$ & $8.6(8.1)$ & $8.9(8.3)$ \\
\hline $\mathrm{I}_{\text {mean }} / \sigma(\mathrm{I})$ & $31.2(19.3)$ & $31.2(18.4)$ & $27.7(6.2)$ \\
\hline Completeness (\%) & $98.0(96.2)$ & 98.0(95.6) & 97.2(99.6) \\
\hline $\mathrm{R}_{\mathrm{sym}}{ }^{2}(\%)$ & $8.7(17.9)$ & $8.0(17.7)$ & $6.1(35.9)$ \\
\hline Phasing Power ${ }^{3}$ & 4.1 & 3.6 & 2.1 \\
\hline
\end{tabular}

${ }^{1}$ Numbers in parentheses indicate values for the highest resolution bin.

$\left.{ }^{2} \mathrm{R}_{\text {sym }}=\sum\left[\mathrm{l}_{\mathrm{i}}-\langle\mathrm{l}\rangle\right](100) / \sum[<\mathrm{l}\rangle\right]$ where $\mathrm{i}$ is ith measurement and $\langle\mathrm{l}\rangle$ is the weighted mean of $\mathrm{I}$.

${ }^{3}$ Phasing power is defined as the mean value of the heavy atom structure factor divided by the lack of closure error.

The overall Figure of Merit was 0.83 .

doi:10.1371/journal.ppat.1002112.t002

search using DaliLite [42] did not identify any proteins of known structure with significant similarity to this C-terminal subdomain (Fig. 2D). As a result, it will be termed the Unique subdomain.

\section{Identification of the receptor binding site}

We sought to identify the details of the interaction between $\mathrm{GspB}_{\mathrm{BR}}$ and the host receptor sialyl-T antigen. Free sialyl-T antigen is a rare reagent that is not commercially available and is challenging to synthesize. Therefore, we developed a 4-step synthesis for $\alpha$-2,3-sialyl (1-thioethyl)galactose (NeuAc $\alpha(2-3)(1-$ $\left.\mathrm{CH}_{3} \mathrm{CH}_{2} \mathrm{~S}\right) \mathrm{Gal} \beta$ ) (see Supporting Protocol S1; Fig. S2) which is the disaccharide truncation of and a synthetic precursor to sialyl$\mathrm{T}$ antigen (NeuAc $\alpha(2-3) \mathrm{Gal} \beta(1-3)$ GalNAc). To confirm that $\alpha$ 2,3-sialyl (1-thioethyl)galactose binds to $\mathrm{GspB}_{\mathrm{BR}}$, we assessed the ability of this disaccharide to inhibit the binding of $S$. gordonii to glycocalicin. In the presence of $44 \mathrm{mM} \alpha$-2,3-sialyl (1-thioethyl)galactose, binding of $S$. gordonii to glycocalicin was reduced by $90 \%$ (Fig. 4A). Given the structural similarity to the native host receptor, this strongly suggests that $\alpha$-2,3-sialyl (1-thioethyl)galactose competes directly for the sialyl-T antigen binding site.

We determined the co-crystal structure of $\alpha$-2,3-sialyl (1thioethyl)galactose in complex with $\mathrm{GspB}_{\mathrm{BR}}$. New electron density consistent with bound disaccharide (Fig. 4B) was apparent within a defined pocket $($ Fig. $4 \mathbf{C}$ ) located at the N-terminus of the $\mathrm{C}$ strand and $\mathrm{C}$-terminus of the $\mathrm{F}$ strand in the Siglec domain (Fig. 3F) of the Siglec subdomain (Fig. 4D). While the similarity of the fold of the Siglec subdomain to mammalian Siglecs might predict a similar binding pocket, both the overall location of the $\alpha$ 2,3-sialyl (1-thioethyl)galactose binding site on the domain and the specific contacts between $\mathrm{GspB}_{\mathrm{BR}}$ to the carbohydrate differ from that of structurally characterized mammalian Siglecs [44-46] (Fig. 5). In fact, in $\mathrm{GspB}_{\mathrm{BR}}$ a helix is positioned in the location where carbohydrates bind to Siglecs, precluding the use of a structurally similar binding site. Instead, the local secondary structure surrounding the $\alpha$-2,3-sialyl (1-thioethyl)galactose binding site resembles a $\beta$-grasp domain, which is a motif that commonly binds the $\alpha$-2,3-linkage of sialic acid based multivalent 
A

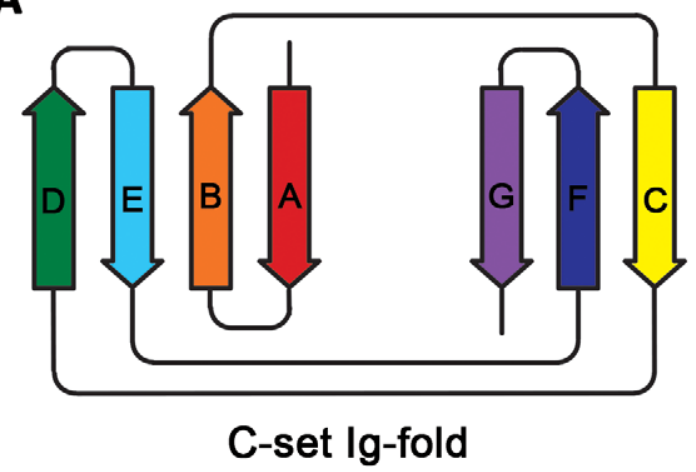

B

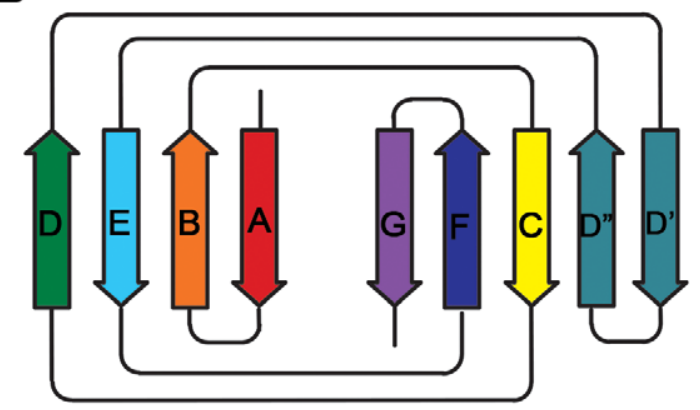

DE-variant C-set Ig-fold

C

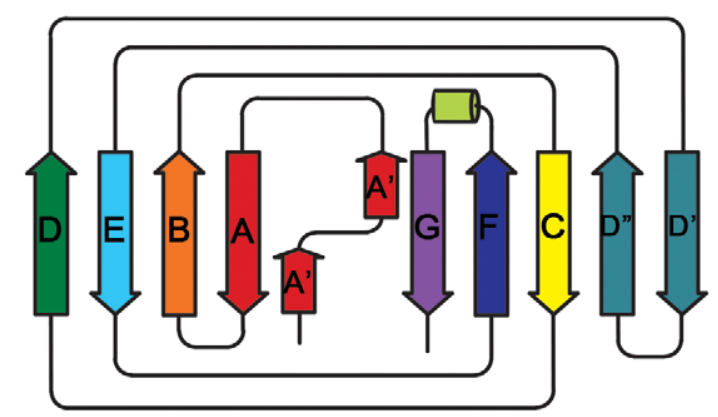

$\mathrm{GspB}_{\mathrm{BR}}$ CnaA Subdomain
D

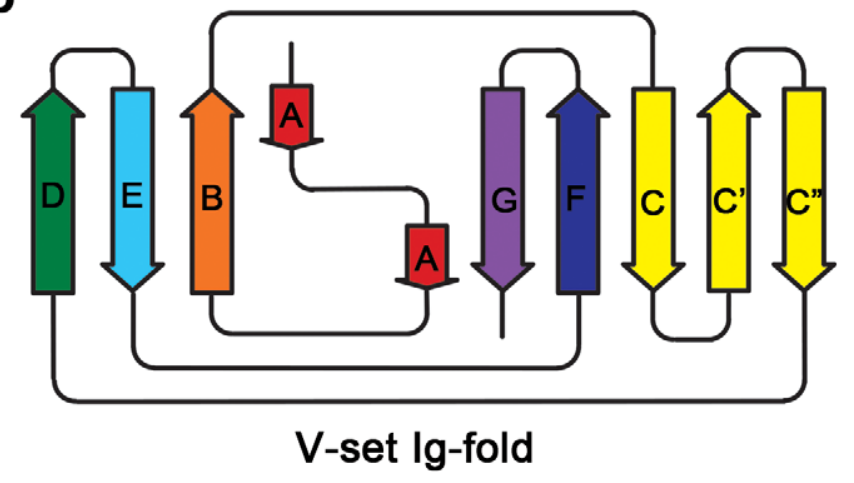

E

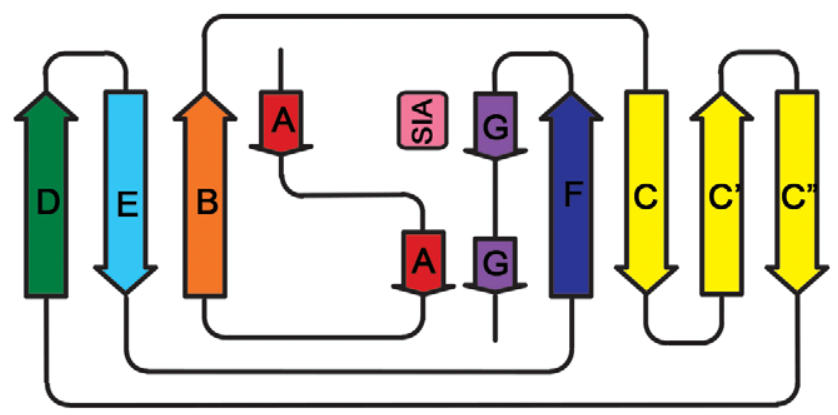

Siglec V-set Ig-fold

$\mathbf{F}$

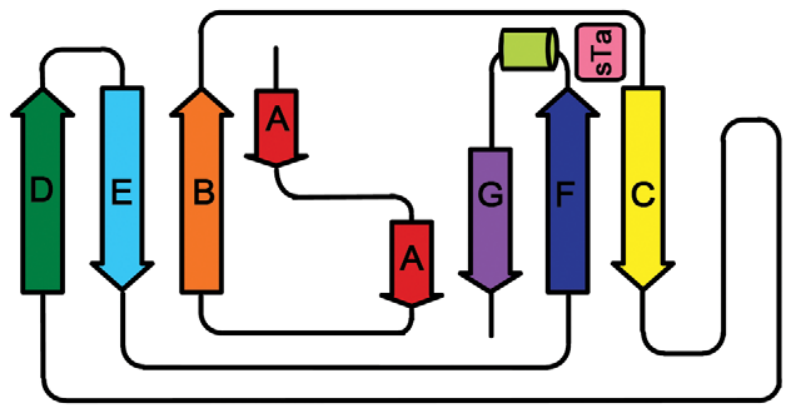

$\mathrm{GspB}_{\mathrm{BR}}$ Siglec Subdomain

Figure 3. Ig-fold topologies. A. Topology diagram of a C-set Ig-fold. B. Topology diagram of the DE-variant of a C-set Ig-fold identified in MSCRAMMs [36]. C. Topology diagram of the $\mathrm{GspB}_{B R} \mathrm{CnaA}_{\mathrm{A}}$ subdomain. D. Topology diagram of a V-set Ig-fold. E. Topology diagram of a Siglec [45] showing the binding location of the sialylated carbohydrate in pink. F. Topology diagram of the $\mathrm{GspB}_{\mathrm{BR}}$ Siglec subdomain showing the binding location of the sialyl-T antigen in pink.

doi:10.1371/journal.ppat.1002112.g003

carbohydrates [47-49]. In $\mathrm{GspB}_{\mathrm{BR}}$, the backbone carbonyls of

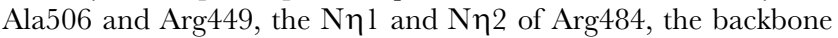
amide nitrogen of Thr483 and the side chain hydroxyl of Tyr443 make direct contacts to the $\alpha$-2,3-sialyl (1-thioethyl)galactose disaccharide, while the side chain hydroxyl of Tyr485 makes water-mediated contacts (Fig. 4B, Table 3).

A comparison of the structure of $\mathrm{GspB}_{\mathrm{BR}}$ determined with and without the disaccharide did not show significant structural changes localized within the binding pocket, which suggests that it is preformed. Thus, while the physiologically-relevant receptor trisaccharide sialyl- $T$ antigen is a rare reagent, we can qualitatively suggest its binding location by modeling the third carbohydrate onto $\alpha$-2,3-sialyl (1-thioethyl)galactose. In our model, all three carbohydrates of the host receptor fit into a pre-formed binding pocket on the Siglec subdomain, with the third carbohydrate of sialyl-T antigen extending toward the Unique subdomain (Fig. S3).

While disaccharide binding to $\mathrm{GspB}_{\mathrm{BR}}$ did not appear to induce obvious changes in conformations of side chains within the binding pocket, the interdomain angle between the CnaA and Siglec subdomains unexpectedly straightened by $40^{\circ}$ (Fig. 6; Video S1). The change in orientation between the CnaA and Siglec subdomains involves rotation around a single hinge consisting of residues Lys398-Asp399-Thr400, where the side chain Asp399 is one of the ion coordinating residues.

Functional relevance of the carbohydrate binding site

To confirm that this crystallographically-identified $\alpha$-2,3-sialyl (1-thioethyl)galactose binding site is indeed important for GspB- 


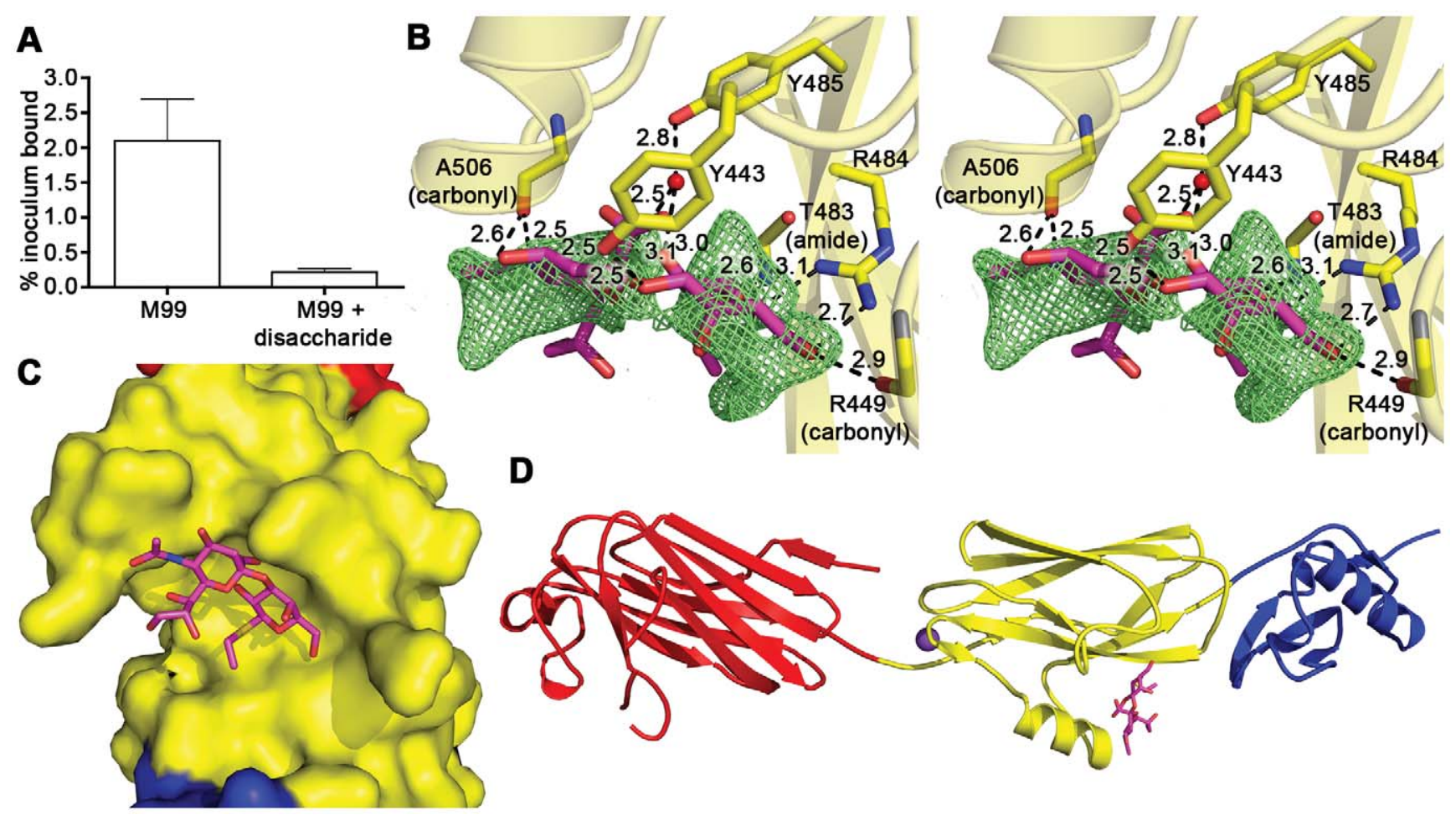

Figure 4. Identification of the receptor binding site. A. Inhibition of S. gordonii strain M99 binding to immobilized glycocalicin by the $\alpha-2,3-$ sialyl (1-thioethyl)galactose disaccharide. Binding was assessed in the absence or presence of $44 \mathrm{mM}$ disaccharide, and is expressed as the percent of input bacteria that remained bound to glycocalicin after repeated washing of the microtiter wells (mean \pm standard deviation). B. Stereo view of the disaccharide binding site superpositioned with $\mathrm{m}\left|\mathrm{F}_{\mathrm{o}}\right|-\mathrm{d}\left|\mathrm{F}_{\mathrm{c}}\right|$ omit electron density (green mesh) calculated in REFMAC5 [65] and contoured to $2.5 \sigma$ after the removal of disaccharide from the model. C. A space filling model of GspB $B_{B R}$ with bound disaccharide (stick model) demonstrates that the carbohydrate receptor binds within a pocket on the surface of the protein. The protein surface is colored by subdomain with the Siglec subdomain in yellow and the Unique subdomain in blue. The stick model of the disaccharide has carbon atoms colored magenta, oxygen atoms colored red, the nitrogen atom colored blue, and the sulfur atom colored yellow. The model is rotated $50^{\circ}$ along the $y$-axis and $40^{\circ}$ along the $x$-axis as compared to panel B. D. Overview of the $\mathrm{GspB}_{\mathrm{BR}}$ structure highlighting the location of the disaccharide binding site in the Siglec subdomain. The model is rotated $90^{\circ}$ along the $\mathrm{x}$-axis as compared to panel $\mathrm{B}$. The disaccharide is shown with magenta bonds and the cation is highlighted as a purple sphere. doi:10.1371/journal.ppat.1002112.g004

mediated binding to GPIb $\alpha$, we generated four isogenic variants of strain M99 with point mutations within the Siglec subdomain of GspB (Table 4). Three of these variants contained mutations within the crystallographically-identified binding site (Y443F, R484E and Y485F), while a control mutation (E401A) was located within the Siglec domain, but away from the receptor binding site. Importantly, none of the point mutations affected surface expression of GspB (Fig. 7A). Each of these isogenic variants had an $86 \%$ or greater decrease in binding to GPIb $\alpha$ in vitro, as compared with the parent strain $(p<0.001)$ (Fig. 7B). This decrease is comparable to that of the $g s p B$ null mutant. In contrast, binding by the E401A variant was not significantly different from that of M99 $(p=0.44)$.

We then selected the R484E mutation for more detailed study. The purified GST-GspB $B_{\mathrm{BR}}$ fusion protein harboring the R484E substitution exhibited a marked decrease in binding to biotinylated sialyl-T antigen (Fig. 7C). The R484E substitution also resulted in reduced binding to human platelets in vitro as assessed by quantifying the amount of input inoculum bound to fixed platelets (Fig. 7D). We next visualized platelets by Differential Interference Contrast (DIC) and fluorescence microscopy and quantified the number of platelets with surface-bound bacteria. In the presence of the DAPI-labeled PS846 (M99 $g s p B)$ or the PS2116 (M99 $g s p b^{\mathrm{R} 484 \mathrm{E}}$ ) strain, substantially fewer platelets had bacteria bound as compared to platelets in the presence of wild-type $S$. gordonii strain M99 (Fig. 8, Table 5). It should be noted that while the platelet numbers were normalized when placed onto the cover slip, during the experiment the wild type bacteria seemed to form microscopic aggregates with the platelets (data not shown) but this was not observed when platelets were mixed with strains PS2116 or PS846. This confirms the importance of this residue for carbohydrate binding.

\section{Impact of Siglec-mediated binding on virulence}

It has previously been demonstrated that loss of GspB expression results in a marked decrease in virulence, as measured by a rat model of infective endocarditis [18]. To assess whether binding via the Siglec subdomain to host carbohydrate receptors contributes to virulence, we examined the impact of the R484E substitution on the ability of S. gordonii strain M99 to produce endocardial infection. We first compared the relative virulence of M99 with strain PS2116, (M99 gspb $\left.{ }^{\mathrm{R} 484 \mathrm{E}}\right)$. Catheterized rats were simultaneously infected intravenously with $2 \times 10^{-5}$ CFU of M99 and PS2116. After $72 \mathrm{~h}$, the animals were sacrificed and the relative levels of bacteria within tissues determined. Animals coinfected with M99 and PS2161 (which carries the spec resistance marker just upstream of $g s p B$ ) served as controls. When assessed at the above time-point, animals co-infected with M99 and PS2116 had significantly reduced densities of the mutant strain within vegetations (mean \pm S.D. $=6.81 \pm 1.70 \log _{10} \mathrm{CU} / \mathrm{g}$. veg.) as compared with parental strain M99 (7.47 $1.69 \log _{10}$ CFU/g. veg; $P<0.02)$. Loss of sialyl-T antigen binding was also associated with 
A

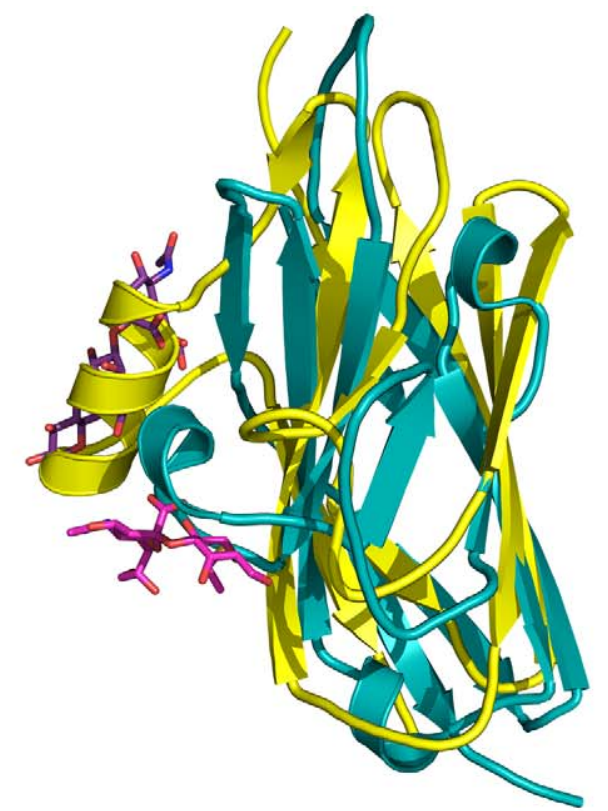

B

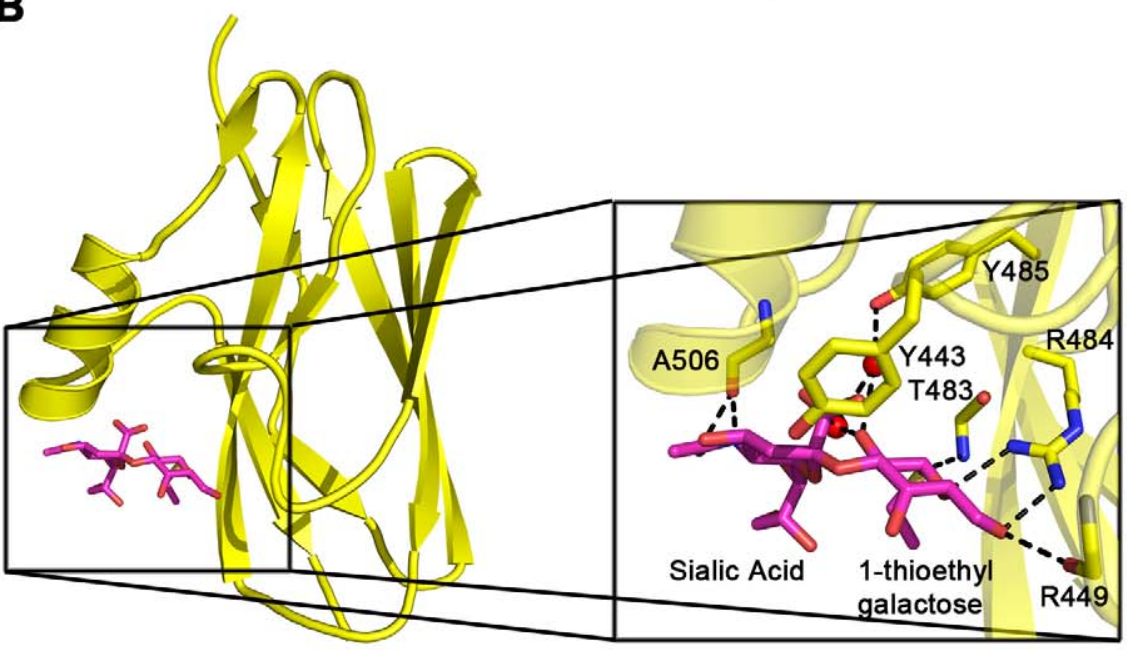

C

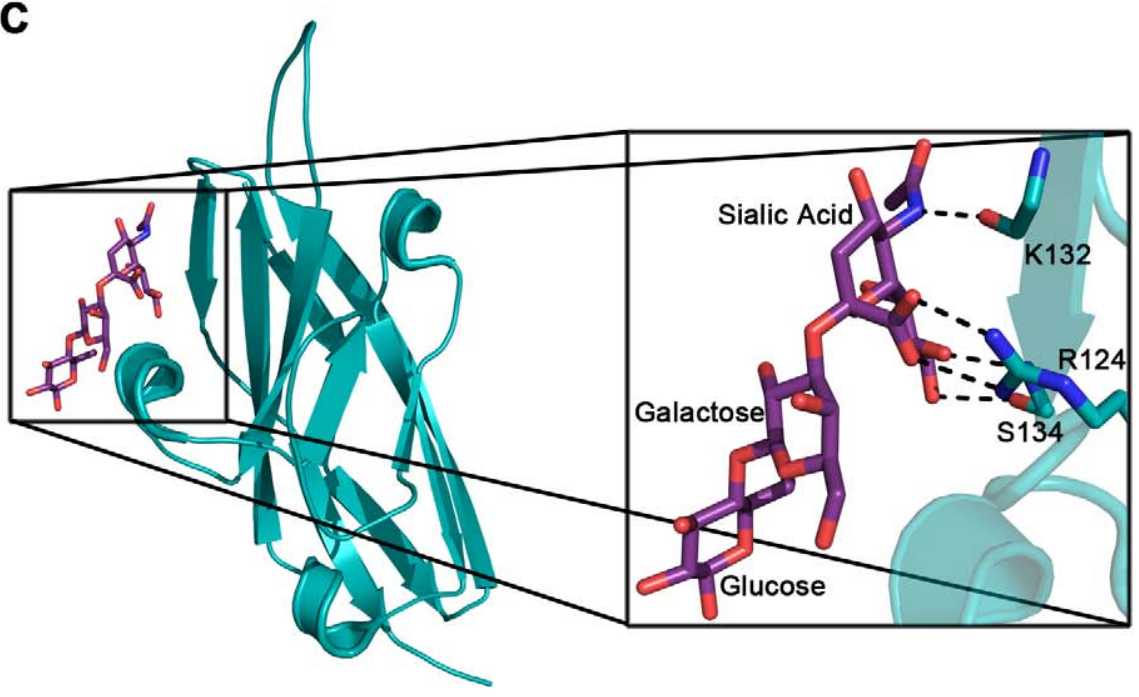


Figure 5. Comparison of carbohydrate binding in the Siglec subdomain of $\mathbf{G s p B}_{\mathbf{B R}}$ and Siglec-5. A. Overlay of the Siglec subdomain of $\mathrm{GspB}_{\mathrm{BR}}$ and Siglec-5. Siglec-5 is colored teal with the carbons of $3^{\prime}$ sialyllactose colored purple. The Siglec subdomain of GspB $B_{\mathrm{BR}}$ is colored yellow with the carbons of $\alpha-2,3$-sialyl (1-thioethyl)galactose colored in magenta. In this panel, the Siglec carbohydrate binding site is occupied by a helix in the $\mathrm{GspB}_{\mathrm{BR}}$ Siglec subdomain therefore eliminating the possibility that this could be a second binding site for $\mathrm{GspB}_{\mathrm{BR}}$. $\mathrm{B} \& \mathrm{C}$. Comparison of the location and details of the carbohydrate binding site in (B) $\mathrm{GspB}_{\mathrm{BR}}$ with that of (C) Siglec-5 (PDB entry 2ZG3; [45]). The Siglec subdomain of GspB $B_{B R}$ is colored yellow with the carbons of $\alpha$-2,3-sialyl (1-thioethyl)galactose colored in magenta and Siglec-5 is colored teal with the carbons of $3^{\prime}$ sialyllactose colored purple. While Siglec-5 is used as an example, it is noted that for all structurally characterized Siglecs (Siglec-1 (aka sialoadhesin); PDB entry 1QFO; [44]), Siglec-5 (2ZG3; [45]), and Siglec-7 (PDB entry 2HRL; [74])), the sialic acid binding pocket is found at the same location. doi:10.1371/journal.ppat.1002112.g005

significantly reduced bacterial densities within kidneys and spleens $(P<0.02$ and $P<0.001$, respectively; Table 6). In parallel studies, no differences were observed between the M99 and the control strain (PS2161), as measured by CFU per gram of tissue within vegetations, kidneys, or spleens (data not shown). We also analyzed these findings by calculating a competition index, in which the ratio of M99 and PS2116 within tissues was normalized for the CFU of each strain within the inoculum. When analyzed by this approach, the densities of the GspB mutant strain PS2116 remained significantly reduced in all tissues, as compared with M99 $(P<0.002)$, while the densities of the control strain were statistically similar.

We then compared the relative virulence of PS2116 with PS846 $(\mathrm{M} 99 \Delta g s p B)$. Infective endocarditis was produced as above, using an inoculum containing the two strains in a $1: 1$ ratio. When assessed at $72 \mathrm{~h}$ post-infection, PS2116 and PS846 had similar densities of organisms within all tissues (Table 6). Of note, the levels of both strains with vegetations were markedly lower than those achieved by wild-type M99 in the above studies. Thus, these two strains appeared comparably attenuated in the setting of endocarditis, indicating that platelet binding by the Siglec domain may be the predominant $\mathrm{GspB}$ interaction contributing to virulence in endocarditis.

\section{The binding regions of SRR adhesins have a modular organization}

While overall sequence analysis of members of the SRR family has identified unifying sequence trends (Fig. 1), sequence comparisons of the binding regions have shown little homology. A structural comparison between the three distinct subdomains of $\mathrm{GspB}_{\mathrm{BR}}$ (Fig. 2A) and the two distinct subdomains of the SRR adhesin Fapl [43] immediately indicates that these family

Table 3. Contacts at the receptor binding site.

\begin{tabular}{|c|c|c|}
\hline Ligand Atom & Protein Atom & Distance $(\AA)$ \\
\hline OAW & Ala506 O & 2.6 \\
\hline NAO & Ala506 O & 2.5 \\
\hline OAT & $\mathrm{HOH} 213^{*}$ & 2.5 \\
\hline OAP & HOH213* & 3.1 \\
\hline $\mathrm{O} 2$ & HOH213* & 2.5 \\
\hline $\mathrm{O} 2$ & $\mathrm{HOH} 199 *$ & 2.9 \\
\hline O3 & Tyr443 OH & 2.5 \\
\hline SAC & Thr483 N & 2.6 \\
\hline O5 & Arg484 N 11 & 3.1 \\
\hline 06 & Arg484 N12 & 2.7 \\
\hline 06 & Arg449 O & 2.9 \\
\hline
\end{tabular}

PLoS Pathogens | www.plospathogens.org

members adopt unrelated folds in their binding domains. Like $\mathrm{GspB}_{\mathrm{BR}}$, Fapl $_{\mathrm{NR}}$ appears to be composed of independently-folded subdomains; however its binding region only contains two modules whereas the binding region of $\mathrm{GspB}_{\mathrm{BR}}$ contains three. Intriguingly, Fapl $1_{\mathrm{NR}}$ contains a helical subdomain at its $\mathrm{N}$ terminus $\left(\operatorname{Fapl}_{\mathrm{NR}}-\alpha\right)$ that does not resemble any of the subdomains of $\mathrm{GspB}_{\mathrm{BR}}$, and a CnaA subdomain at its C-terminus $\left(\mathrm{Fapl}_{\mathrm{NR}}-\beta\right)$ that resembles the $\mathrm{N}$-terminal $\mathrm{CnaA}$ subdomain of $\mathrm{GspB}_{\mathrm{BR}}$ $\left(\mathrm{GspB}_{\mathrm{BR}-\mathrm{C}}\right)$. This suggested to us that members of the SRR family could undergo reorganization of the modules within their host binding regions, with particular modules or combinations of modules conferring specific properties.

Accordingly, we re-analyzed the binding regions of selected members of the family using a new strategy, where we used BLAST [50] and ClustalW [51] to query the sequences of the binding regions of SRR adhesins with input sequences corresponding to subdomains of either $\mathrm{GspB}_{\mathrm{BR}}$ or Fapl $\mathrm{l}_{\mathrm{NR}}$, or of short regions ( $\sim 200$ amino acids) of sequences of structurally uncharacterized binding regions of SRR adhesins. These modified sequence comparisons strongly suggest that the binding regions of members of the SRR family have indeed evolved to contain modules (Fig. 9), and show several distinct groupings. For example, all three assessed SRR adhesins with carbohydrate binding partners contain the Siglec and Unique subdomains in tandem, strongly suggesting that the inclusion of these modules within the binding region confers lectin-like binding characteristics.

These focused sequence alignments additionally identify that a CnaA subdomain may be common in SRR adhesins, appearing in three of the eight binding regions that we assessed. As opposed to the combination of the Siglec and Unique subdomains, which are associated with carbohydrate binding, each adhesin containing a CnaA subdomain may have a different binding specificity (Fig. 9). Interestingly, in the SRR adhesins that were assessed, the CnaA subdomains were always found paired with sequence for another subdomain.

It is important to note that several of the binding regions of the SRR adhesins do not have significant sequence identity to any protein of known structure. For example, the binding region of $S$. agalactiae SRR1 ( $\left.\mathrm{SRR} l_{\mathrm{GBS}}\right)$ likely contains two subdomains, $\mathrm{SRR} 1_{\mathrm{GBS}}-\alpha$ at the N-terminus, which does not contain detectable sequence similarity to any protein of known fold, and SRR $1_{\text {GBS }}-\beta$ at the C-terminus, which has sequence similarity to MSCRAMMs and likely adopts a similar folding topology to CnaA. Likewise, no part of the binding regions of S. pneumoniae PsrP, S. aureus SraP, or $S$. epidermidis $\mathrm{seSRR}_{\mathrm{BR}}$ exhibits detectable sequence similarity to any known protein, but the latter two have high sequence similarity to each other.

\section{Discussion}

SRR adhesins have been identified in a variety of Gram-positive pathogens, and have been implicated as virulence factors in a wide spectrum of infections $[5,6,12,18]$. The diversity of these infections (e.g., endocarditis, meningitis, pneumonia) and the broad scope of 


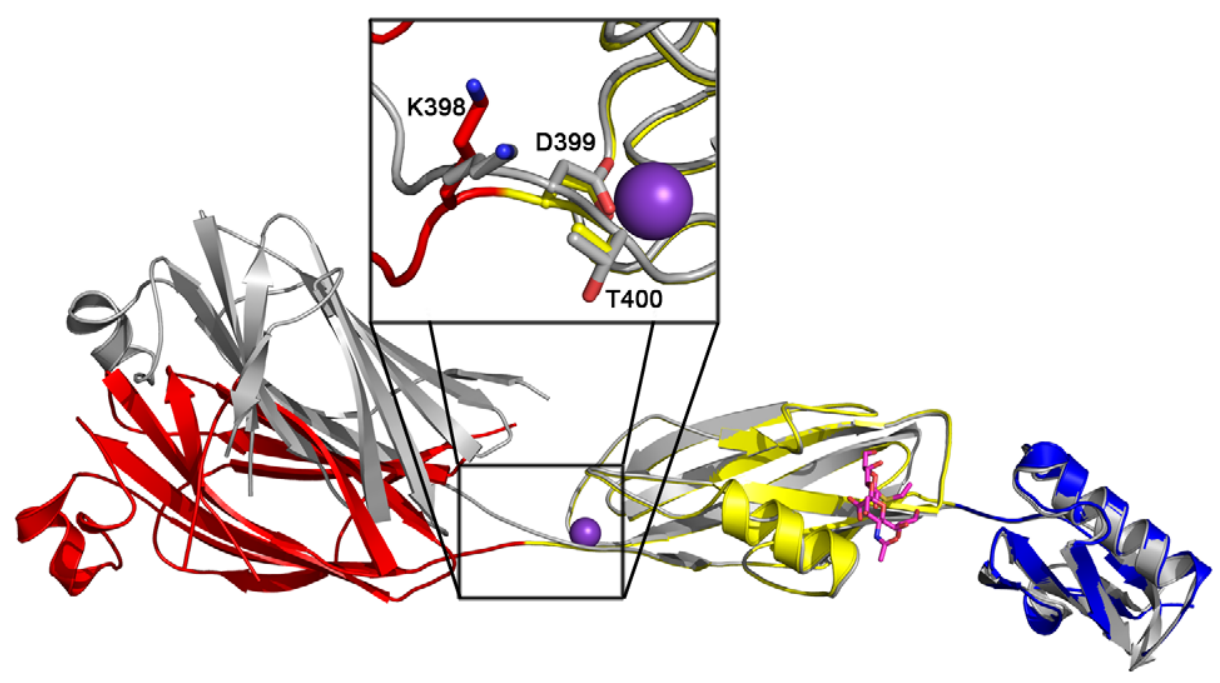

Figure 6. Interdomain Angle. Differences in interdomain angles between structures of $\mathrm{GspB}_{\mathrm{BR}}$. The structures of the Siglec and Unique subdomains of unbound and disaccharide-bound $\mathrm{GspB}_{\mathrm{BR}}$ were aligned using Dyndom [69]. The unbound structure is colored in silver, and the structure in complex with $\alpha$-2,3-sialyl (1-thioethyl) galactose is colored by subdomain with the CnaA subdomain in red, the Siglec subdomain in yellow and the Unique subdomain colored in blue, and cation colored purple. The side chains of hinge residues Lys398, Asp399, and Thr400 are highlighted in the boxed inset. The difference in interdomain angle between the two structures is a $40^{\circ}$. A movie morphing these states is seen in Video $\mathbf{S 1}$. doi:10.1371/journal.ppat.1002112.g006

their anatomic locations are consistent with the binding regions of the SRR proteins differing considerably in their selectivity. Indeed, although only a few ligands for this family of proteins have been identified, they range from carbohydrates (such as sialyl-T antigen) [32] to proteins (such as keratins) [16,17]. To date, however, the structural basis for this selectivity has been unknown.

In several members of the family (e.g. the SRR adhesins of $S$. gordonii and $S$. pneumoniae) the domain between the two SRR regions mediates binding. However, for many of these SRR adhesins (i.e. SraP of $S$. aureus), the host receptor has yet to be identified [6,43], and the delineation of the binding region is assumed based upon sequence comparisons within the family. Among the best characterized of the SRR adhesins is GspB from $S$. gordonii, which has demonstrated binding affinity for the sialyl-T antigen carbohydrate decorating platelet glycoprotein GPIb $\alpha$. Our crystal structure of the binding region, $\mathrm{GspB}_{\mathrm{BR}}$, identified a modular organization with three subdomains (Fig. 2A), two of which are organized around Ig-folds. Proteins containing Ig-folds are commonly found within the mammalian immune system, where they exhibit a variety of functions; however, Ig-folds are not uncommon within pathogens, where they act exclusively as virulence factors. The first characterized bacterial protein to contain an Ig-fold was PapD, a chaperone for the assembly of pili in E. coli [37]. The Ig-fold has been observed in several other types of virulence factors, including components of pili (carbohydratebinding fimbriae) [41,52], and in adhesins of varying specificity for host receptors, including MSCRAMMs [34-36] and invasins [3840].

Co-crystallization of $\mathrm{GspB}_{\mathrm{BR}}$ with $\alpha$-2,3-sialyl (1-thioethyl)galactose identified a specific binding site for this disaccharide within the Siglec subdomain (Fig. 4B-D), and the introduction of single point mutations within this region significantly reduced levels of binding of $S$. gordonii to glycocalicin. Moreover, the R484E substitution in GspB reduced platelet binding by M99 (Fig. 7D, Fig. 8, Table 5), and had a marked reduction in binding of the GST-GspB $B_{\mathrm{BR}}$ fusion protein to sialyl-T antigen (Fig. $\mathbf{7 C}$ ), confirming the importance of the Siglec subdomain for carbohydrate binding. These results indicate that this binding pocket within the Siglec subdomain is required for binding to sialyl-T antigen, and that the interaction of this GspB subdomain with sialyl-T antigen is important for binding to platelets.

When examined in a co-infection model of infective endocarditis, the isogenic variant of M99 expressing GspB-R484E (strain PS2116) was also significantly reduced in virulence (most notably a $78 \%$ reduction in bacterial levels within vegetations as compared with the parent strain). When PS2116 was tested with PS846 $(\mathrm{M} 99 \Delta g s p B)$ in this model, the strains had comparable but reduced densities within target tissues (as compared with WT M99), indicating that they were similarly reduced in virulence. These findings indicate that the predominant property of GspB contributing to the pathogenesis of endocarditis is its interaction with sialyl-T antigen. In previous in vivo studies, co-infection with M99 and PS846 yielded more pronounced differences between the strains, as measured by densities of organisms within target tissues [18]. This may reflect subtle experimental differences, such as inoculum size, but could also be due to residual binding activity of the mutated GspB. Of note, PS2116 had low but detectable levels of platelet binding in vitro. Similarly, the GST-GspB ${ }_{\mathrm{BR}}-\mathrm{R} 484 \mathrm{E}$ had measurable levels of binding to sialyl- $\mathrm{T}$ antigen, as compared to GST alone. This residual binding seen in vitro, presumably due to the other key residues identified by crystallography, may account for the residual virulence of the PS2116 strain. Alternatively, although ligands for GspB other than sialyl-T antigen have not been identified, it is conceivable that GspB has other interactions

Table 4. Strain names with descriptions.

\begin{tabular}{ll}
\hline Strain name & Description \\
\hline M99 & S. gordonii strain M99 wild-type \\
PS2116 & S. gordonii strain M99 gsp $B^{\text {R484E }}$ \\
PS846 & S. gordonii strain M99 $\Delta s p B$ \\
PS2161 & 5'gspB::spec control strain \\
\hline doi:10.1371/journal.ppat.1002112.to04
\end{tabular}


A

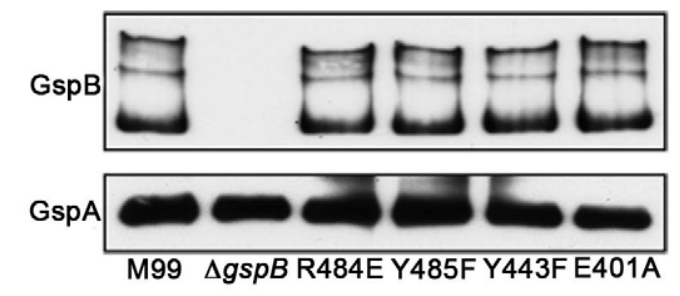

B

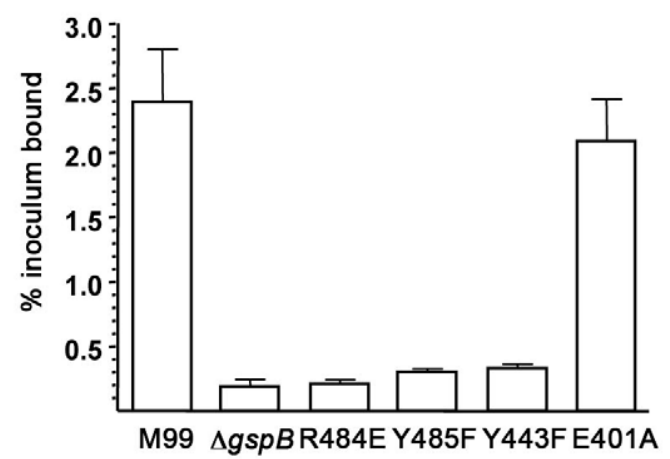

C

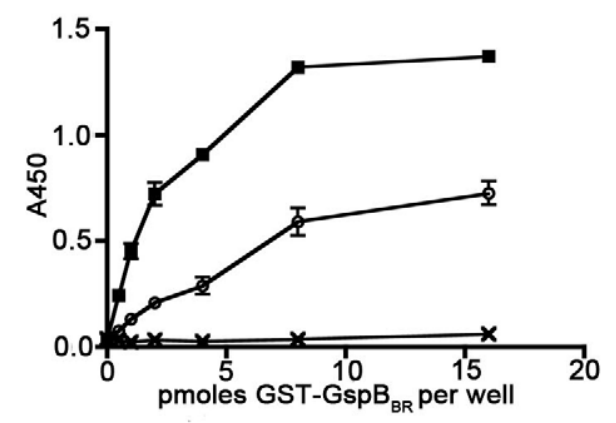

D

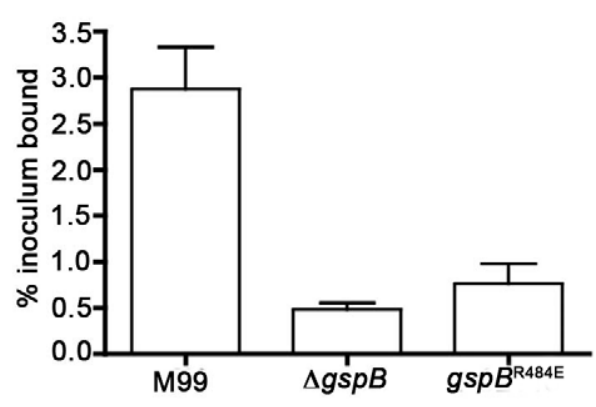

Figure 7. Amino acid substitutions in the carbohydrate binding site of $\mathbf{G s p B}_{\mathbf{B R}}$. A. Expression of GspB and variant proteins on the $S$. gordonii cell surface. Peptidoglycan-linked proteins were extracted from the bacterial cell surface and analyzed by western blotting using a polyclonal anti-GspB serum (upper panel). The nitrocellulose membrane was then re-probed using a polyclonal anti-GspA serum (lower panel) in order to assess the relative efficiency of protein extraction and protein loading (GspA is another LPXTG-linked protein that is expressed by $S$. gordonii M99). B. Binding of S. gordonii strain M99 and derivative strains to glycocalicin immobilized in microtiter wells. Binding is expressed as the percent of input bacteria that remained bound to glycocalicin after repeated washing of the wells (mean \pm standard deviation). The labels of each column indicate the sequence of the protein expressed in $S$. gordonii, with M99 indicating wild type, $\triangle g s p B$ indicating a strain where the gene encoding GspB has been deleted, and remaining columns indicating the amino acid substitution present in the protein. E401 is a control residue not located near the binding pocket. C. Binding of sialyl-

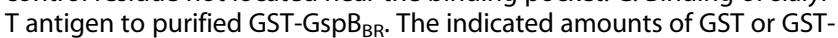
$\mathrm{GspB}_{\mathrm{BR}}$ wild-type (wt) and variant (R484E) proteins were immobilized in microtiter wells, and the binding of biotinylated sialyl-T antigen to each protein was detected by using peroxidase-conjugated streptavidin along with a chromogenic peroxidase substrate. Binding is expressed as the mean \pm standard deviation $(n=3)$. The o represents purified GST$\mathrm{GspB}_{\mathrm{BR}}-\mathrm{R} 484 \mathrm{E}$, the $\mathrm{x}$ represents purified GST, and the represents purified GST-GspB $B_{B R}$. D. Binding of M99 and derivative strains to human platelets. Binding is expressed as the percent of input bacteria that remained bound to platelets after repeated washing of the wells (mean \pm standard deviation).

doi:10.1371/journal.ppat.1002112.g007

in vivo that contribute to virulence in this setting, beyond those mediating platelet binding.

Upon binding of the $\alpha$-2,3-sialyl (1-thioethyl)galactose to $\mathrm{GspB}_{\mathrm{BR}}$, a $40^{\circ}$ interdomain angle straightening is observed between the CnaA and Siglec subdomains (Fig. 6, Video S1). The interdomain hinge is centered at the ion binding site, making the interdomain angle change reminiscent of calcium dependent interdomain angle changes observed in C-cadherin, which also contains modules of Ig-fold topology [53]. There are several possibilities for the origin and role of this interdomain reorganization in $\mathrm{GspB}_{\mathrm{BR}}$. It is first possible that $\mathrm{GspB}_{\mathrm{BR}}$ exhibits natural flexibility between these two domains, and that the difference in crystal contacts artifactually resulted in the straighter form of the protein being trapped in the co-structure of $\mathrm{GspB}_{\mathrm{BR}}$ with $\alpha-2,3$ sialyl (1-thioethyl)galactose. Intriguingly, however, an interdomain straightening is also observed in small angle x-ray scattering (SAXS) of the binding region of SRR protein $\mathrm{Fapl}_{\mathrm{NR}}$ upon lowering the $\mathrm{pH}$ to 5.0, where $\mathrm{Fapl}_{\mathrm{NR}}$ has highest affinity for its host receptor [43]. Those experiments, performed in solution, are not limited by crystal contacts and suggest that interdomain straightening upon ligand binding could be a conserved feature of members of the SRR family. Physiological roles of interdomain straightening upon ligand binding include improved hydrodynamics in the cardiovascular system or oral cavity. The elongation upon ligand binding is perhaps more compellingly reminiscent of the interdomain straightening that occurs upon the binding of Pselectin and $\beta 3$-integrin to their respective ligands [54]. These proteins have been suggested to form "catch bonds" in order to have increased affinity to their ligand upon the application of tensile force. Each of these possibilities for the observed straightening of $\mathrm{GspB}_{\mathrm{BR}}$ upon ligand binding is currently under investigation.

While both Siglecs and $\mathrm{GspB}_{\mathrm{BR}}$ bind to carbohydrate receptors, the structural details of disaccharide binding to this novel bacterial Siglec bear little resemblance to the binding of sialic acid moieties to characterized mammalian Siglecs [44-46] (Fig. 5). Indeed, comparison of the binding sites reveals that the secondary structure of $\mathrm{GspB}_{\mathrm{BR}}$ has a helix that is found at the same location where 3 ' sialyllactose binds to Siglec-5 (Fig. 5A), thus eliminating the possibility of analogous carbohydrate binding. Instead, $\mathrm{GspB}_{\mathrm{BR}}$ appears to use a $\beta$-grasp domain to form the specific host receptor binding site, a domain found in another group of sialic acid binding proteins, staphylococcal superantigen-like proteins (SSLs) that forms a V-like cleft for carbohydrate binding [47-49]. Nevertheless, both mammalian Siglecs and $\mathrm{GspB}_{\mathrm{BR}}$ have binding pockets predominated by tyrosines and arginines. With a closer look at each binding site, in Siglecs, the sialic acid moiety of the binding partner makes a salt bridge with an arginine residue. This salt bridge is not present in the binding site of $\mathrm{GspB}_{\mathrm{BR}}$ despite the presence of R484 and its key role in binding; this arginine instead binds to the C6 hydroxyl and the pyranose oxygen of the 1-thioethylgalactose (Table 3). 
Importantly, the structure of $\mathrm{GspB}_{\mathrm{BR}}$ provides insight into the binding repertoire of other SRR proteins, and specifically highlights which modules mediate binding to host carbohydrate receptors. Homologues of GspB containing regions with high sequence similarity to the Siglec and Unique subdomains (such as S. gordonii strain Challis $\mathrm{Hsa}$ and $S$. sanguinis $\mathrm{SrpA}$ ) are also demonstrated lectins. Supporting this sequence analysis, our cocrystal structure of $\mathrm{GspB}_{\mathrm{BR}}$ with $\alpha$-2,3-sialyl (1-thioethyl)galactose demonstrates that the first two carbohydrates of host receptor bind within a pre-formed pocket on the Siglec subdomain. Using this experimental co-structure as a starting point, we could model binding of the sialyl-T antigen. This model is consistent with the third carbohydrate of this trisaccharide binding to an extended lobe on this pocket. This region of the binding pocket is still within the Siglec subdomain but extends toward the Unique subdomain (Fig. S3).

The structures of both $\mathrm{GspB}_{\mathrm{BR}}$ and $\mathrm{Fapl}_{\mathrm{NR}}$ identified a CnaA-like subdomain within the binding region, and our sequence analysis (Fig. 9) additionally predicts a CnaA subdomain at the C-terminus of the binding region of $S$. agalactiae SRR1 (SRR1 $\left.1_{\mathrm{GBS}} \boldsymbol{\beta}\right)$, an adhesin for keratin 4 [16]. As opposed to the Siglec subdomain, which contains an identifiable receptor binding site, the precise function of the CnaA subdomain is less clear. MSCRAMMs, such as CnaA from $S$. aureus, recognize peptides through the formation of a binding site between two Ig-like domains (Fig. S4). The bound peptide forms an additional strand that becomes a part of the Ig-fold. In contrast, GspB $\mathrm{BR}_{\mathrm{BR}}, \mathrm{Fapl}_{\mathrm{NR}}$, and $\mathrm{SRR} \mathrm{l}_{\mathrm{GBS}}$ each apparently contain a single CnaA subdomain amongst the modules in the binding region (Fig. 9). Our modeling suggests that binding of a peptide between two domains in a manner analogous to binding of a peptide to other MSCRAMMs is not feasible. Indeed, an additional strand found in the subdomain of $\mathrm{GspB}_{\mathrm{BR}}$ occupies the peptide binding site of MSCRAMMs. (Fig. S4, Fig. 3C strand $\mathbf{A}^{\prime}$ ). Nevertheless, in SRR adhesins, the CnaA subdomain is always found together with at least one other subdomain, suggesting that the function may either require or be tuned by the presence of a second subdomain. Indeed, recent studies on the binding region of Fapl support this hypothesis. The structures of Fap $1_{N R}-\alpha$ (helical subdomain) and Fapl $1_{N R}-\beta$ (CnaA subdomain) identified a region of surface-exposed hydrophobic residues on each domain that is predicted to be contiguous based upon SAXS. Fapl normally mediates binding of this bacterium to the oral cavity, and mutagenesis of these hydrophobic residues abrogated binding to an in vitro tooth model consisting of saliva-coated hydroxylapatite [43].

Our sequence analysis additionally predicts that the binding regions of other SRR adhesins contain modules that are structurally distinct from those identified in either $\mathrm{GspB}_{\mathrm{BR}}$ or Fap $1_{\mathrm{NR}}$. Two of these, $S$. aureus $\mathrm{SraP}_{\mathrm{BR}}$ and $S$. epidermidis $\mathrm{SeSRR}_{\mathrm{BR}}$, have high sequence identity to each other, but no detectable sequence similarity to any other SRR adhesin (Fig. 9). This strongly suggests that they bind a common, as yet unidentified, host receptor that is distinct from the carbohydrates and keratins recognized by other SRR adhesin family members. By comparison, neither S. pneumoniae $\mathrm{PsrP}_{\mathrm{BR}}$, which has been demonstrated to bind keratin 10, nor the N-terminal subdomain of $S$. agalactiae SRR $1_{\mathrm{BR}}$, which binds keratin 4, have detectable sequence similarity to any currently available sequence (Fig. 9). Given this analysis, it is clear that a more detailed understanding of the binding characteristics of the SRR adhesin family will require that for each module type, the binding partner should be identified, and the structure of the binding region should be determined both alone and in complex with the appropriate host receptor.
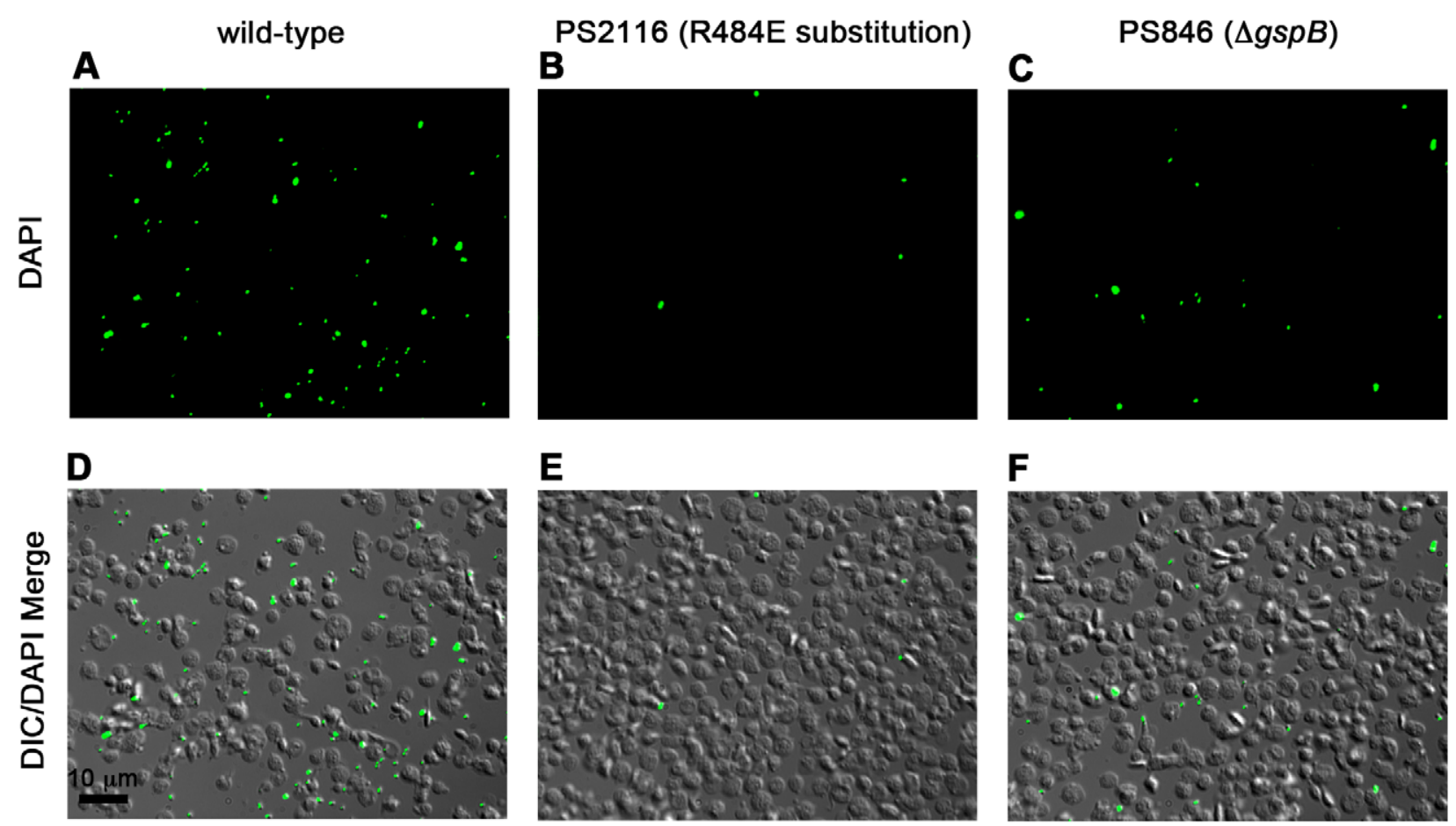

Figure 8. Binding of wild type S. gordonii strain M99, PS2116 (strain M99 with gspb harboring the R484E substitution), and PS846 $(\Delta g s p B)$ to platelets. Fixed platelets were adhered to poly-L-lysine coated cover slips, quenched, and bound to live bacteria stained with DAPI. A-C. Fluorescent images of each strain with DAPI stain. D-F. Merge between the DIC image and the DAPI image to show relative number of platelets. doi:10.1371/journal.ppat.1002112.g008 
Table 5. Binding of three variants of S. gordonii to human platelets.

\begin{tabular}{ll}
\hline Strain & \% of Platelets with Bacteria Bound (Mean \pm S.D.) \\
\hline M99 & $32.0 \pm 8.7$ \\
PS2116 & $3.0 \pm 1.7^{*}$ \\
PS846 & $7.0 \pm 1.7^{*}$ \\
\hline
\end{tabular}

${ }^{*} p=0.01$ as compared with M99.

Note: The difference in binding between the PS2116 and PS846 strains were not statistically significant.

doi:10.1371/journal.ppat.1002112.t005

\section{Materials and Methods}

\section{Ethics statement}

All procedures involving rats were approved by the Los Angeles Biomedical Research Institute animal use and care committee, following the National Institutes of Health guidelines for animal housing and care. Platelets were collected using a protocol approved by the UCSF Committee on Human Research (H1 193-25513-07) and by the VUMC Human Research Protection Program IRB Committee (110364).

\section{Protein expression and purification}

The DNA encoding residues 233-617 of GspB ( $\left.\mathrm{GspB}_{\mathrm{BR}}\right)$ was cloned into the pGEX vector containing an N-terminal glutathione S-transferase (GST) fusion tag as described [11]. This clone contains a single base pair change as compared to the deposited NCBI sequence that results in a serine at position 444 instead of an asparagine. The protein was expressed with E. coli BL21 Gold (Stratagene) in Luria Broth Medium as described [55] and purified using a GST affinity column (GE Healthcare). The GST tag was removed from $\mathrm{GspB}_{\mathrm{BR}}$ using Factor $\mathrm{Xa}$, and $\mathrm{GspB}_{\mathrm{BR}}$ was further purified using size exclusion chromatography on a Superdex 200 10/300 GL column (GE Healthcare) with buffer containing $20 \mathrm{mM}$ Tris pH 7.4 [55].

\section{Crystallization}

All crystals of $\mathrm{GspB}_{\mathrm{BR}}$ were grown using the hanging drop vapor diffusion technique at $23^{\circ} \mathrm{C}$ [55] using $1 \mu \mathrm{l}$ protein solution and $1 \mu \mathrm{l}$ of reservoir solution equilibrated against $1 \mathrm{ml}$ of the reservoir solution. Crystals of native $\mathrm{GspB}_{\mathrm{BR}}$ grew from two chemically distinct sets of conditions. The first set of conditions included $\mathrm{GspB}_{\mathrm{BR}}$ at a concentration of $6 \mathrm{mg} / \mathrm{ml}$ buffered in $20 \mathrm{mM}$ Tris $\mathrm{pH} 7.4$ and equilibrated against a reservoir solution containing 33\% Jeffamine ED-2001, 0.1 M HEPES pH 7.5 and $0.15 \mathrm{M} \mathrm{KCl}$ at $23^{\circ} \mathrm{C}$. These crystals belonged to the primitive orthorhombic space group $P 2{ }_{1} 2_{1} 2_{1}$ with unit cell dimensions $\mathrm{a}=33.7 \AA, \mathrm{b}=96.8 \AA, \mathrm{c}=100.2 \AA$ with $\alpha=\beta=\gamma=90^{\circ}$. Prior to data collection, crystals were cryo-protected in a solution containing all of the chemical components of each reservoir solution and $15 \%$ glycerol then flash-cooled in liquid nitrogen. The dataset used for refinement merged to $1.4 \AA$ resolution.

The second type of native $\mathrm{GspB}_{\mathrm{BR}}$ crystals grew when the protein was equilibrated against a reservoir solution containing $25 \%$ polyethylene glycol 3350, 0.1 M HEPES pH 7.5, 0.15 M $\mathrm{NH}_{4} \mathrm{CH}_{3} \mathrm{COO}$, and $10 \mathrm{mM}$ spermidine and induced crystal growth from $10 \mathrm{mg} / \mathrm{ml} \mathrm{GspB}_{\mathrm{BR}}$ buffered in $20 \mathrm{mM}$ HEPES pH 7.5 at $18^{\circ} \mathrm{C}$. These crystals also belonged to the orthorhombic space group $P 2{ }_{1} 2_{1} 2_{1}$, but had altered unit cell dimensions of $\mathrm{a}=33.4 \AA, \mathrm{b}=86.7 \AA, \mathrm{c}=117.9 \AA, \alpha=\beta=\gamma=90^{\circ}$. Prior to data collection, these crystals were flash cooled in liquid nitrogen without additional cryo-protectant. The best dataset from this crystal form merged to $2.0 \AA$ resolution.

Crystals of $\mathrm{GspB}_{\mathrm{BR}}$ in complex with the $\alpha$-2,3-sialyl (1thioethyl)galactose disaccharide were grown using the hanging drop vapor diffusion method with $6 \mathrm{mg} / \mathrm{mL} \mathrm{GspB}_{\mathrm{BR}}$ in buffer containing $1 \mathrm{mM} \alpha$-2,3-sialyl (1-thioethyl)galactose and $20 \mathrm{mM}$ Tris $\mathrm{pH}$ 7.4. The reservoir solution contained 8\% PEG 3350, $7.5 \mathrm{mM} \mathrm{CoCl}_{2}, 7.5 \mathrm{mM} \mathrm{NiCl}, 7.5 \mathrm{mM} \mathrm{CdCl}_{2}, 7.5 \mathrm{mM} \mathrm{MgCl}_{2}$, and $0.1 \mathrm{M}$ HEPES $\mathrm{pH}$ 7.5. Crystals grew within two days and were cryo-protected in reservoir solution supplemented with $20 \%$ glycerol and flash-cooled in liquid nitrogen. The disaccharide cocrystals formed in the primitive monoclinic space group $P 2_{1}$ with unit cell dimensions $\mathrm{a}=69.9 \AA, \mathrm{b}=34.0 \AA, \mathrm{c}=83.4 \AA$ with $\alpha=\gamma=90^{\circ}$ and $\beta=99.2^{\circ}$. The best data from this crystal form merged to 1.9 Å resolution.

\section{Data collection and processing}

Crystals were assessed for diffraction quality at the Stanford Synchrotron Radiation Lightsource (SSRL) beamlines 9-2, 11-1, and 12-2 and the Life Sciences Collaborative Access Team (LSCAT) beamlines ID-21-D/F/G. Datasets were collected using the beamlines, temperatures, wavelengths, and detectors listed in Table 1 and Table 2. All data were processed using the HKL2000 [56] and CCP4 [57] suites of programs.

\section{Preparation of heavy atom derivatives and structure determination}

A Dy ${ }^{3+}$ derivative was prepared by soaking pre-formed crystals of $\mathrm{GspB}_{\mathrm{BR}}$ in $1 \mathrm{mM} \mathrm{DyCl}$ for three days. Data were collected at three wavelengths near the $\mathrm{Dy}^{3+} \mathrm{L} 3$ edge (Table 2). $\mathrm{Dy}^{3+}$ bound to a single site in the protein as determined using the SHELXD [58] subroutine in the program SHARP [59]. While $\mathrm{HoCl}_{3}$ also successfully derivatized $\mathrm{GspB}_{\mathrm{BR}}$, non-isomorphism between crys-

Table 6. Effect of Sialyl-T antigen binding on virulence in a rat model of infective endocarditis.

\begin{tabular}{|c|c|c|c|c|}
\hline \multirow[t]{2}{*}{ Strain pairs } & \multirow[t]{2}{*}{ No. of animals } & \multicolumn{3}{|c|}{ S. gordonii densities (Mean \pm S.D. $\log _{10}$ CFU/g tissue) } \\
\hline & & Vegetations & Kidneys & Spleen \\
\hline M99 (wt) & & $7.47 \pm 1.69$ & $3.72 \pm 0.72$ & $3.77 \pm 0.66$ \\
\hline PS2116 (R484E) & 11 & $6.81 \pm 1.70^{*}$ & $3.40 \pm 0.86^{*}$ & $3.20 \pm 0.72^{* *}$ \\
\hline PS846 ( $\Delta g s p B)$ & & $5.76 \pm 2.15$ & $2.72 \pm 1.32$ & $3.32 \pm 1.10$ \\
\hline PS2116 (R484E) & 7 & $5.45 \pm 2.69$ & $2.53 \pm 1.12$ & $2.92 \pm 0.88$ \\
\hline
\end{tabular}


A

\begin{tabular}{|c|c|c|c|c|c|c|c|c|}
\hline $\mathrm{GspB}_{\mathrm{BR}-\mathrm{C}}$ & & & $18 / 56$ & & & $15 / 43$ & & -- \\
\hline GspB BR-su & & & & $31 / 46$ & $34 / 51$ & & -- & \\
\hline Fap1 $_{N R}-\beta$ & & & $24 / 54$ & & & -- & & $15 / 43$ \\
\hline Hsa & & & & $51 / 65$ & -- & & $34 / 51$ & \\
\hline SrpA & & & & -- & $51 / 65$ & & $31 / 46$ & \\
\hline $\mathrm{SRR}_{\mathrm{GBS}^{-}} \beta$ & & & -- & & & $24 / 54$ & & $18 / 56$ \\
\hline SraP & $62 / 75$ & -- & & & & & & \\
\hline \multirow[t]{2}{*}{ seSRR } & -- & $62 / 75$ & & & & & & \\
\hline & seSRR & SraP & $\mathrm{SRR} 1_{\mathrm{GBS}}-\beta$ & SrpA & Hsa & Fap $1_{N R}-\beta$ & $\mathrm{GspB}_{\mathrm{BR}-\mathrm{su}}$ & $\mathrm{Gsp}_{\mathrm{BR}-\mathrm{C}}$ \\
\hline
\end{tabular}

B

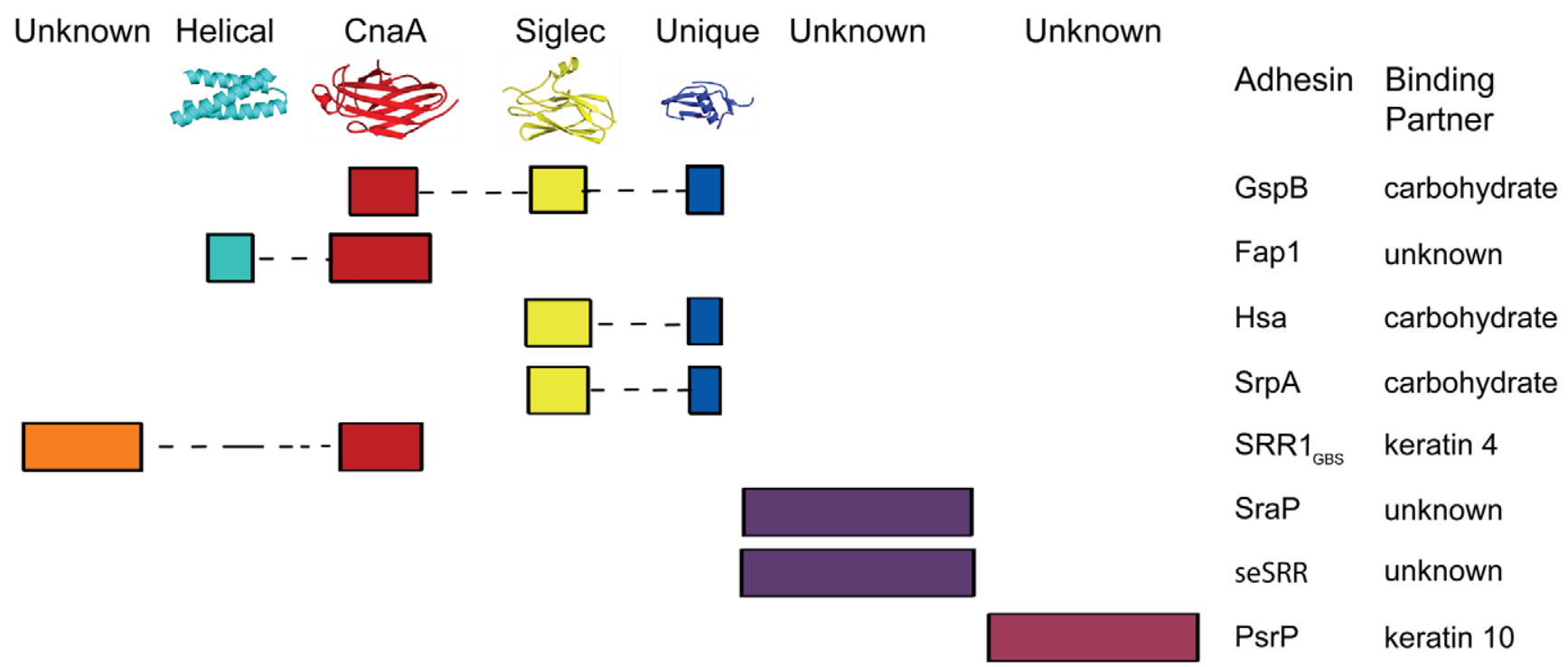

Figure 9. Modular organization of the binding regions within adhesins of the SRR superfamily. A. A summary of BLAST [50] or ClustalW [51] sequence alignments of the binding regions of SRR adhesins. The first number indicates sequence identity and the second indicates sequence similarity. $\mathrm{GspB}_{\mathrm{BR}}$ has been divided into its subdomains, with $\mathrm{GspB}_{\mathrm{BR}-\mathrm{C}}$ indicating the $\mathrm{CnaA}$ subdomain and $\mathrm{GspB}_{\mathrm{BR}-\mathrm{su}}$ indicating both the Siglec and Unique subdomains. Only the C-terminal subdomain of $S R R 1_{G B S}\left(S R R 1_{G B S}-\beta\right)$ and Fap $1_{N R}\left(F a p 1_{N R}-\beta\right)$ are used in this analysis since the $N$-terminal domains do not share detectable sequence identity with other sequences used in this analysis. Boxes in grey indicate SRR pairs that do not have detectable sequence identity within the binding region. B. A schematic summarizing (A) The colored rectangles represent similar regions of sequence, and are drawn to scale. If known, representative structures of each domain are illustrated along the top, and binding partners are indicated along the side.

doi:10.1371/journal.ppat.1002112.g009

tals prevented the use of this second derivative in a traditional MIR calculation. The non-isomorphism was so severe that phases calculated in SHARP [59] only used data from a single $\mathrm{DyCl}_{3}$ soaked crystal and did not include a native dataset for reference. This process resulted in reasonable phasing statistics and an overall figure of merit of 0.83 at $2.0 \AA$ resolution (Table 2). Phases were improved by solvent flattening in DM [60] which produced electron density maps of high quality (Fig. 2B). Automated chain tracing was performed using PHENIX [61], which was able to trace residues 251-316 and 327-601, representing $94.7 \%$ of the model. This resulted in an initial $R_{\text {cryst }}$ of $23.8 \%$ and $R_{\text {free }}$ of $25.5 \%$.

The lack of isomorphism between crystals prevented transfer of these initial coordinates to other data sets using a simple rigid body refinement. As a result, the model from the $\mathrm{Dy}^{3+}$ data set was transferred to the remaining data sets using the program PHASER [62] followed by rigid body refinement in CNS [63]. Each structure was subjected to alternate rounds of model building using the program COOT [64] and refinement using CNS [63] and REFMAC [65].
The coordinates for the $\alpha$-2,3-sialyl (1-thioethyl)galactose were built using CCP4i Sketcher [66] and PRODRG [67]. Refinement statistics for all final models are listed in Table 1. Figures were created using PyMOL [68], and inter-domain rotations were determined using DynDom [69].

\section{Synthesis of the $\alpha-2,3$-sialyl (1-thioethyl)galactose} disaccharide

Based upon work by Danifshesky and coworkers [70], we developed a four-step synthesis for the sialyl-T antigen precursor, $\boldsymbol{\alpha}$-2,3-sialyl (1-thioethyl)galactose (see Supporting Protocol S1). The correct synthesis of the disaccharide was verified by NMR (Fig. S2). The $\alpha$-2,3-sialyl (1-thioethyl)galactose was resuspended in water for all applications.

\section{Site-directed Mutagenesis}

Point mutations of $g s p B$ were introduced into the $S$. gordonii chromosome via a strategy that involved recombination by double cross-over between $g_{s p} B$ codons 487-602 and a gene approxi- 
mately $300 \mathrm{bp}$ upstream. This approach ensured incorporation of only the intended mutation of $\operatorname{ssp} B$ codons ranging from 399 to 485 , and avoided possible imprecise recombination within the SRR regions. As a first step, the $5^{\prime}$ end of $g s p B$ (codons 1 to 486 along with $200 \mathrm{nts}$ from a non-coding region upstream) was replaced with a chloramphenicol resistance cassette as follows. A $0.5 \mathrm{~kb}$ segment of a gene of unknown function upstream of $g s p B$ was amplified using PCR. The product was digested with XhoI and ClaI and then cloned upstream of the cat gene in pC326 [71]. A segment spanning gsp B codons 487 to 602 was then amplified using primers $\mathrm{B} 487 \mathrm{~F}$ and $\mathrm{B} 602 \mathrm{R}$, digested with SpeI and NotI, and cloned downstream of the cat gene. The resulting plasmid, pC326 $\Delta 5^{\prime}$ B, was used to transform $S$. gordonii strain M99 as described [4]. One of the chloramphenicol-resistant transformants, (M99 $\Delta 5^{\prime}$ gspB::cat), was selected for subsequent gene replacement.

A series of plasmids was then constructed to facilitate the replacement of the $5^{\prime}$ end of $g s p B$ in M99 $\Delta 5^{\prime}$ gsp $B:: c a t$. The XhoI$\mathrm{ClaI}$ fragment from pC326 $\Delta 5^{\prime} \mathrm{B}$ was cloned upstream of the spec gene in $\mathrm{pS} 326$ [72], and a $1 \mathrm{~kb}$ NsiI-SpeI fragment of gsp $B$ (spanning codons 1 to 296), was cloned downstream. A SpeI-NotI fragment spanning codons 296 to 602 was then cloned downstream of the NsiI-SpeI fragment. Point mutations in the resulting plasmid, pS326B602, were generated by a two-stage PCR procedure. In the first stage, primer $25 \mathrm{~F}$ along with a reverse $g s p B$ primer, or the corresponding $g s p B$ forward primer along with primer B602R, were used to amplify the upstream or downstream segments, respectively. The two PCR products were combined for the second stage and then amplified using primers $25 \mathrm{~F}$ and B602R. The PGR product was digested with SpeI and NotI and then used to replace the corresponding segment of pS326B602. The incorporation of only the intended change in any segment generated by PCR was confirmed by DNA sequence analysis. Plasmids were then used to transform M99 $\Delta 5^{\prime}$ gsp B::cat, resulting in a replacement of $\Delta 5^{\prime} g s p B:: c a t$ with a $5^{\prime} g s p B:: s p e c$ variant. As a control, the wild-type $g s p B$ sequence along with the spec cassette (pS326B602) was also crossed into the M99 $\Delta 5^{\prime}$ gspB::cat chromosome (generating strain PS2161). Transformants were selected on spectinomycin and scored for the loss of chloramphenicol resistance. Expression of the variant GspB proteins on the bacterial cell surface was verified by western blotting as described [72].

\section{$\mathrm{GspB}_{\mathrm{BR}}$ binding to biotinylated sialyl-T antigen}

GST, GST-GspB BR and GST-GspB $B_{B R}-R 484 E$ were purified from E. coli as described [55]. The purified proteins were diluted to $320 \mu \mathrm{M}$ into DPBS, serial two-fold dilutions were made, and $50 \mu \mathrm{l}$ of each dilution was added to wells of a 96-well microtiter plate. After incubating the plate overnight at $4^{\circ} \mathrm{C}$, unbound proteins were removed by aspiration and wells were rinsed with $100 \mu \mathrm{l}$ DPBS. Biotinylated sialyl-T antigen (sialyl-T antigen conjugated to biotin via a polyacrylamide linker; GlycoTech Corporation) was diluted to $50 \mu \mathrm{g} / \mathrm{ml}$ in DPBS containing $1 \times$ Blocking Reagent (Roche), $50 \mu \mathrm{l}$ was added to each well, and the plate was incubated for $2 \mathrm{~h}$ at RT with vigorous rocking. After removing unbound biotin-sialyl-T antigen, wells were rinsed three times with $100 \mu \mathrm{l}$ DPBS, $50 \mu \mathrm{l}$ of streptavidin-conjugated horseradish peroxidase $(0.1 \mu \mathrm{g} / \mathrm{ml}$ in DPBS $)$ was added to each well and the plate was incubated for $1 \mathrm{~h}$ at $23^{\circ} \mathrm{C}$. The wells were washed twice with $100 \mu \mathrm{l}$ DPBS, and then $200 \mu \mathrm{l}$ of a solution of OPD $(0.4 \mathrm{mg} / \mathrm{ml}$ citrate-phosphate buffer) was added to each well. The contents of the wells were mixed by gently vortexing the plate, and the absorbance at $450 \mathrm{~nm}$ was measured $20 \mathrm{~min}$ after the addition of the OPD substrate. Data were plotted as the means \pm standard deviations, with $n=3$.

\section{Binding of S. gordonii to glycocalicin and platelet monolayers}

The binding of $S$. gordonii to immobilized glycocalicin was performed as described previously [32]. In brief, strains were grown for $18 \mathrm{hr}$, washed twice with DPBS, sonicated briefly to disrupt aggregated cells, and then diluted to approximately $2 \times 10^{7}$ per ml. To determine whether $\alpha-2,3$ sialyl (1-thioethyl)galactose inhibited binding to glycocalicin, the washed and sonicated bacteria were diluted into DPBS or DPBS containing $44 \mathrm{mM} \alpha$ 2,3-sialyl (1-thioethyl)galactose, $\mathrm{pH}$ 7.5. The bacterial suspensions were then applied to wells of a microtiter plate that had been coated with glycocalicin $(1.25 \mu \mathrm{g} /$ well). After $2 \mathrm{~h}$ at room temperature, the unbound bacteria were removed by aspiration. Wells were washed three times with DPBS, and the bound bacteria were released by trypsinization. The number of input and bound bacteria were determined by plating serial dilutions of the bacterial suspensions on sheep blood agar plates, and binding was expressed as the percent of the input bound to glycocalicin. The binding of $S$. gordonii to immobilized human platelets was assessed as described previously [4]. Results of both assays are reported as the means \pm standard deviations, with $n=6$. Differences in binding were compared by the unpaired $t$-test.

\section{DIC and fluorescence microscopy}

$S$. gordonii strains M99 wild type, PS2116, and PS846 were grown in $5 \mathrm{~mL}$ of Todd Hewitt Broth at $37^{\circ} \mathrm{C}$ without shaking for 18 hours. Cells were then vortexed to resuspend bacteria and spun down at $4000 \times \mathrm{g}$ for ten minutes. The supernatant was removed and the bacteria washed twice with $5 \mathrm{~mL}$ of DPBS containing $\mathrm{MgCl}_{2}$ and $\mathrm{CaCl}_{2}$. The bacteria were then resuspended in $5 \mathrm{~mL}$ of DPBS and sonicated briefly to disrupt aggregated clumps. To $1 \mathrm{~mL}$ of each cell suspension, $500 \mathrm{nM} \mathrm{4',6-diamidino-2-pheny-}$ lindole (DAPI) was added.

Platelets were fixed using $1.6 \%$ paraformaldehyde. $500 \mu \mathrm{L}$ of platelets were mounted on poly-L-lysine coated cover slips (in a 6 well tray) which was spun at $400 \times \mathrm{g}$ for ten minutes in order to promote platelet adherence to the cover slips. Excess platelets were removed by washing with Tris Buffered Saline (TBS) and $1 \mathrm{~mL}$ of TBS was added to each well. $500 \mu \mathrm{L}$ of the bacterial suspension was added to the platelets and the samples were rocked vigorously for 30 minutes at $23^{\circ} \mathrm{C}$. Excess bacteria were then removed by washing three times with TBS. Each sample was mounted onto a slide for microscopy. Slides were imaged using Nikon TiE Inverted light microscope equipped with a Photometrics CoolSnap HQ CCD camera. Platelets were imaged with a $100 \times 1.49$ NA objective using DIC optics and a DAPI filter cube. Image $\mathrm{J}$ software was used to create the contrast and composite images.

\section{Rat model of infective endocarditis}

A competition model of infective endocarditis was produced in Sprague-Dawley female rats (250-300 g) as described previously [18]. In brief, the animals were anesthetized with ketamine (35 mg/ $\mathrm{kg})$ and xylazine $(10 \mathrm{mg} / \mathrm{kg})$. A sterile polyethylene catheter was surgically placed across the aortic valve of each animal, such that the tip was positioned in the left ventricle. Catheters were left in place throughout the study. Catheterized animals were then infected intravenously (IV) with an inoculum containing $2 \times 10^{5} \mathrm{CFU}$ of both strains (i.e., a 1:1 mixture of i) $S$. gordonii strain M99 and strain PS2116 (5'gsp $\left.B^{R 484 E}:: s p e c\right)$, ii) M99 and strain PS2161 (5' gspB::spec control strain), or iii) PS2116 and strain PS846 (M99 $\Delta s s p B:$ :pEVP3; $\mathrm{Cm}^{\mathrm{R}}$ ) [18,73]. At $72 \mathrm{~h}$ post-infection, animals were sacrificed with thiopental (100 mg, intraperitoneally). Animals were included in the final analysis only if the catheters were correctly positioned across 
the aortic valve at the time of sacrifice, and if macroscopic vegetations were seen. All cardiac vegetations, as well as samples of the kidneys and spleens were harvested, weighed, homogenized in saline, serially diluted, and plated onto Todd Hewitt agar (THA) (for the parental S. gordonii strain M99) and THA containing $100 \mu \mathrm{g} / \mathrm{ml}$ of spectinomycin (for strains PS2116 and PS2161) or chloramphenicol $5 \mathrm{mg} / \mathrm{ml}$ (for strain PS846) for quantitative culture, to determine the number of $\mathrm{CFU} / \mathrm{g}$ of $S$. gordonii strains within tissues. After $48 \mathrm{~h}$ of incubation at $37^{\circ} \mathrm{C}$, bacterial colonies were counted. The number of bacteria within tissues was expressed as the $\log _{10}$ CFU per gram of tissue. Differences between means were compared for statistical significance by the paired $t$-test, using $p \leq 0.05$ as the threshold for significance. The data were also analyzed by calculating a "competition index," which was defined as the ratio of S. gordonii strain M99 and strain PS2116 or PS2161, as well as PS2116 and PS846, within tissues for each animal, normalized by the ratio of organisms in the inoculum. The mean of the $\log _{10}$ normalized ratios was tested against the hypothesized 'no effect' mean value of 0 , as described previously, using a paired $t$-test.

\section{Accession numbers}

The coordinates and structure factors for S. gordonii strain M99 $\mathrm{GspB}_{\mathrm{BR}}$ have been deposited in the Research Collaboratory for Structural Bioinformatics Protein Data Bank with accession codes 3QG5 (native 1), 3QG6 (native 2, crystal form 2), and 3QD1 ( $\alpha$ 2,3-sialyl (1-thioethyl)galactose bound).

\section{Supporting Information}

Figure S1 The cation binding site. Stereo view of the cation binding site superpositioned with $2 \mathrm{~m}\left|\mathrm{~F}_{\mathrm{o}}\right|-\mathrm{d}\left|\mathrm{F}_{\mathrm{c}}\right|$ electron density calculated in REFMAC5 [65] and contoured to $1.5 \sigma$. Carbons are shown in grey, oxygens are shown in red, nitrogens are shown in blue and the cation is shown as a purple sphere. Bond distances are shown in $\AA$.

(TIF)

Figure S2 ${ }^{1} \mathrm{H} \mathrm{NMR}, 400 \mathrm{MHz}\left(\mathrm{CDCl}_{3}\right)$ spectrum of $\boldsymbol{\alpha}$-2,3-sialyl (1-thioethyl)galactose.

(TIF)

Figure S3 Predicted binding of sialyl-T antigen to the receptor binding pocket. Using the co-structure of $\mathrm{GspB}_{\mathrm{BR}}$ with $\alpha$-2,3-sialyl (1-thioethyl)galactose as a starting point, we developed a model for binding of sialyl-T antigen to the Siglec subdomain. Coordinates for sialyl-T antigen were prepared in COOT [64] by manually linking the 3-position of $\mathrm{N}$-acetylgalactosamine to the 1-position of the galactose in $\alpha$-2,3-sialyl (1-thioethyl) galactose and removing the thioethyl group. The position of the $\alpha$-2,3-sialyl (1-thioethyl) galactose group was fixed to its position in the experimentallydetermined structure and the galactosamine manually optimized

\section{References}

1. Takahashi Y, Konishi K, Cisar JO, Yoshikawa M (2002) Identification and characterization of hsa, the gene encoding the sialic acid-binding adhesin of Streptococcus gordonii DL1. Infect Immun 70: 1209-1218.

2. Handley PS, Correia FF, Russell K, Rosan B, DiRienzo JM (2005) Association of a novel high molecular weight, serine-rich protein $(\mathrm{SrpA})$ with fibril-mediated adhesion of the oral biofilm bacterium Streptococcus cristatus. Oral Microbiol Immunol 20: 131-140.

3. Wu H, Mintz KP, Ladha M, Fives-Taylor PM (1998) Isolation and characterization of Fapl, a fimbriae-associated adhesin of Streptococcus parasanguis FW213. Mol Microbiol 28: 487-500.

4. Bensing BA, Sullam PM (2002) An accessory sec locus of Streptococcus gordonii is required for export of the surface protein GspB and for normal levels of binding to human platelets. Mol Microbiol 44: 1081-1094.

5. Sanchez CJ, Shivshankar P, Stol K, Trakhtenbroit S, Sullam PM, et al. (2010) The pneumococcal serine-rich repeat protein is an intra-species bacterial to avoid steric clashes. In this model, all three sugars bind within a contiguous pre-formed pocket on the surface of the protein between the Siglec and Unique subdomains. The Siglec subdomain is colored yellow, the Unique subdomain is colored blue, and the modeled sialyl-T antigen is colored with gray carbons. (TIF)

Figure S4 Binding of MSCRAMMs to fibrinogen receptors. The interdomain peptide binding observed in SdrG (PDB entry 1R17) uses a "dock, lock, and latch" mechanism of fibrinogen recognition [34] is unlikely to be used by SRR adhesins. This interdomain mechanism requires two adjacent Ig-fold domains that create a binding site; the fibrinogen peptide binds between these two domains. Modeling additional interdomain angles in $\mathrm{GspB}_{\mathrm{BR}}$ suggests that the short linker region between the CnaA and Siglec subdomains will prevent the interdomain angle from becoming sufficiently acute to allow the Siglec subdomain to act as a surrogate second subdomain. In addition, $\mathrm{GspB}_{\mathrm{BR}}$ contains an additional strand at the N-terminus of the CnaA subdomain (Fig. 3C strand $\mathbf{A}^{\prime}$ ) that occupies the peptide binding site of MSCRAMMs. Unbound SdrG is colored gray and bound SdrG is colored red with the fibrinogen peptide colored cyan.

(TIF)

Video S1 Range of interdomain angles observed in crystals of $\mathrm{GspB}_{\mathrm{BR}}$. The movie morphs between states observed in the crystal structures and was produced using LSQMAN [75] and PyMOL [68]. The most extended conformation was observed in the costructure of $\mathrm{GspB}_{\mathrm{BR}}$ with the $\alpha$-2,3-sialyl (1-thioethyl)galactose disaccharide.

(MP4)

Test S1 Results and discussion regarding the cation binding site. (DOCX)

Protocol S1 Identification of the cation binding site and synthesis of $\alpha$-2,3-sialyl (1-thioethyl)galactose.

(DOG)

\section{Acknowledgments}

We thank Dr. Mikio Tanabe, Dr. Timothy Panosian, Dr. Jessica Vey, Rebecca McRae, Dr. Matthew Duvernay, Jessica Mazerick, Dr. Rajalakshmi Nambiar, Laura Anzaldi, Summer Young, Tarjani Thaker, and Dr. Kathryn McCulloch for experimental assistance.

\section{Author Contributions}

Conceived and designed the experiments: GG GAS MJT PMS TMI. Performed the experiments: TMP BAB YQX BJM VY KMO MJT. Analyzed the data: TMP BAB YQX BJM TMT NJW MJT TMI. Contributed reagents/materials/analysis tools: VY. Wrote the paper: TMP BAB YQX BJM GAS MJT PMS TMI.

adhesin that promotes bacterial aggregation in vivo and in biofilms. PLoS Pathog 6(8): e1001044. doi:10.1371/journal.ppat.1001044.

6. Siboo IR, Chambers HF, Sullam PM (2005) Role of SraP, a Serine-Rich Surface Protein of Staphylococcus aureus, in binding to human platelets. Infect Immun 73: 2273-2280.

7. Seifert KN, Adderson EE, Whiting AA, Bohnsack JF, Crowley PJ, et al. (2006) A unique serine-rich repeat protein (Srr-2) and novel surface antigen (epsilon) associated with a virulent lineage of serotype III Streptococcus agalactiae. Microbiology 152: 1029-1040.

8. Wu H, Fives-Taylor PM (1999) Identification of dipeptide repeats and a cell wall sorting signal in the fimbriae-associated adhesin, Fapl, of Streptococcus parasanguis. Mol Microbiol 34: 1070-1081.

9. Plummer C, Wu H, Kerrigan SW, Meade G, Cox D, et al. (2005) A serine-rich glycoprotein of Streptococcus sanguis mediates adhesion to platelets via GPIb. Br J Haematol 129: 101-109. 
10. Takamatsu D, Bensing BA, Prakobphol A, Fisher SJ, Sullam PM (2006) Binding of the streptococcal surface glycoproteins GspB and Hsa to human salivary proteins. Infect Immun 74: 1933-1940.

11. Takamatsu D, Bensing BA, Cheng H, Jarvis GA, Siboo IR, et al. (2005) Binding of the Streptococcus gordonii surface glycoproteins GspB and Hsa to specific carbohydrate structures on platelet membrane glycoprotein Ib alpha. Mol Microbiol 58: 380-392.

12. van Sorge NM, Quach D, Gurney MA, Sullam PM, Nizet V, et al. (2009) The group B streptococcal serine-rich repeat 1 glycoprotein mediates penetration of the blood-brain barrier. J Infect Dis 199: 1479-1487.

13. Mistou MY, Dramsi S, Brega S, Poyart C, Trieu-Cuot P (2009) Molecular dissection of the secA2 locus of group B Streptococcus reveals that glycosylation of the Srrl LPXTG protein is required for full virulence. J Bacteriol 191: 4195-4206.

14. Yajima A, Urano-Tashiro Y, Shimazu K, Takashima E, Takahashi Y, et al. (2008) Hsa, an adhesin of Streptococcus gordonii DL1, binds to alpha2-3-linked sialic acid on glycophorin A of the erythrocyte membrane. Microbiol Immunol 52: 69-77.

15. Takahashi Y, Sandberg AL, Ruhl S, Muller J, Cisar JO (1997) A specific cell surface antigen of Streptococcus gordonii is associated with bacterial hemagglutination and adhesion to alpha2-3-linked sialic acid-containing receptors. Infect Immun 65: 5042-5051.

16. Samen U, Eikmanns BJ, Reinscheid DJ, Borges F (2007) The surface protein Srr-1 of Streptococcus agalactiae binds human keratin 4 and promotes adherence to epithelial HEp-2 cells. Infect Immun 75: 5405-5414.

17. Shivshankar P, Sanchez C, Rose LF, Orihuela CJ (2009) The Streptococcus pneumoniae adhesin PsrP binds to Keratin 10 on lung cells. Mol Microbiol 73: 663-679.

18. Xiong YQ, Bensing BA, Bayer AS, Chambers HF, Sullam PM (2008) Role of the serine-rich surface glycoprotein GspB of Streptococcus gordonii in the pathogenesis of infective endocarditis. Microb Pathog 45: 297-301.

19. Obert C, Sublett J, Kaushal D, Hinojosa E, Barton T, et al. (2006) Identification of a Candidate Streptococcus pneumoniae core genome and regions of diversity correlated with invasive pneumococcal disease. Infect Immun 74: 4766-4777.

20. Petersen HJ, Keane C, Jenkinson HF, Vickerman MM, Jesionowski A, et al. (2010) Human platelets recognize a novel surface protein, PadA, on Streptococcus gordonii through a unique interaction involving fibrinogen receptor GPIIbIIIa. Infect Immun 78: 413-422.

21. Kerrigan SW, Jakubovics NS, Keane C, Maguire P, Wynne K, et al. (2007) Role of Streptococcus gordonii surface proteins $\mathrm{SspA} / \mathrm{SspB}$ and $\mathrm{Hsa}$ in platelet function. Infect Immun 75: 5740-5747.

22. Jakubovics NS, Kerrigan SW, Nobbs AH, Stromberg N, van Dolleweerd CJ, et al. (2005) Functions of cell surface-anchored antigen I/II family and Hsa polypeptides in interactions of Streptococcus gordonii with host receptors. Infect Immun 73: 6629-6638.

23. Ganesh VK, Rivera JJ, Smeds E, Ko YP, Bowden MG, et al. (2008) A structural model of the Staphylococcus aureus ClfA-fibrinogen interaction opens new avenues for the design of anti-staphylococcal therapeutics. PLoS Pathog 4: e1000226.

24. McDevitt D, Nanavaty T, House-Pompeo K, Bell E, Turner N, et al. (1997) Characterization of the interaction between the Staphylococcus aureus clumping factor (ClfA) and fibrinogen. Eur J Biochem 247: 416-424.

25. Miajlovic H, Loughman A, Brennan M, Cox D, Foster TJ (2007) Both complement- and fibrinogen-dependent mechanisms contribute to platelet aggregation mediated by Staphylococcus aureus clumping factor B. Infect Immun 75: 3335-3343.

26. Loughman A, Fitzgerald JR, Brennan MP, Higgins J, Downer R, et al. (2005) Roles for fibrinogen, immunoglobulin and complement in platelet activation promoted by Staphylococcus aureus clumping factor A. Mol Microbiol 57: 804-818.

27. O'Brien L, Kerrigan SW, Kaw G, Hogan M, Penades J, et al. (2002) Multiple mechanisms for the activation of human platelet aggregation by Staphylococcus aureus: roles for the clumping factors ClfA and ClfB, the serine-aspartate repeat protein SdrE and protein A. Mol Microbiol 44: 1033-1044.

28. Fitzgerald JR, Foster TJ, Cox D (2006) The interaction of bacterial pathogens with platelets. Nat Rev Microbiol 4: 445-457.

29. Seo HS, Xiong YQ Mitchell J, Seepersaud R, Bayer AS, et al. (2010) Bacteriophage lysin mediates the binding of streptococcus mitis to human platelets through interaction with fibrinogen. PLoS Pathog 6(8): e1001047. doi:1001010.1001371/journal.ppat.1001047.

30. Durack DT (1975) Experimental bacterial endocarditis. IV. Structure and evolution of very early lesions. J Pathol 115: 81-89.

31. Yajima A, Takahashi Y, Konishi K (2005) Identification of platelet receptors for the Streptococcus gordonii DL1 sialic acid-binding adhesin. Microboil Immunol 49: 795-800.

32. Bensing BA, Lopez JA, Sullam PA (2004) The Streptococcus gordonii surface proteins GspB and Hsa mediate binding to sialylated carbohydrate epitopes on the platelet membrane glycoprotein Ib alpha. Infect Immun 72: 6528-6537.

33. Takahashi Y, Takashima E, Shimazu K, Yagishita H, Aoba T, et al. (2006) Contribution of sialic acid-binding adhesin to pathogenesis of experimental endocarditis caused by Streptococcus gordonii DL1. Infect Immun 74: 740-743.

34. Ponnuraj K, Bowden MG, Davis S, Gurusiddappa S, Moore D, et al. (2003) A "dock, lock, and latch" structural model for a staphylococcal adhesin binding to fibrinogen. Cell 115: 217-228.
35. Symersky J, Patti JM, Carson M, House-Pompeo K, Teale M, et al. (1997) Structure of the collagen-binding domain from a Staphylococcus aureus adhesin. Nat Struct Biol 4: 833-838.

36. Deivanayagam CC, Wann ER, Chen W, Carson M, Rajashankar KR, et al. (2002) A novel variant of the immunoglobulin fold in surface adhesins of Staphylococcus aureus: crystal structure of the fibrinogen-binding MSCRAMM, clumping factor A. EMBO J 21: 6660-6672.

37. Holmgren A, Branden CI (1989) Crystal structure of chaperone protein PapD reveals an immunoglobulin fold. Nature 342: 248-251.

38. Cota E, Jones C, Simpson P, Altroff H, Anderson KL, et al. (2006) The solution structure of the invasive tip complex from Afa/Dr fibrils. Mol Microbiol 62: 356-366.

39. Hamburger ZA, Brown MS, Isberg RR, Bjorkman PJ (1999) Crystal structure of invasin: a bacterial integrin-binding protein. Science 286: 291-295.

40. Jedrzejczak R, Dauter Z, Dauter M, Piatek R, Zalewska B, et al. (2006) Structure of DraD invasin from uropathogenic Escherichia coli: a dimer with swapped beta-tails. Acta Crystallogr D Biol Crystallogr 62: 157-164.

41. Vengadesan K, Ma X, Dwivedi P, Ton-That H, Narayana SV (2011) A Model for Group B Streptococcus Pilus Type 1: The Structure of a 35-kDa C-Terminal Fragment of the Major Pilin GBS80. J Mol Biol 407: 731-743.

42. Holm L, Rosenstrom P (2010) Dali server: conservation mapping in 3D. Nucleic Acids Res 38: W545-549.

43. Ramboarina S, Garnett JA, Zhou M, Li Y, Peng Z, et al. (2010) Structural insights into serine-rich fimbriae from gram-positive bacteria. J Biol Chem 285 : 32446-32457.

44. May AP, Robinson RC, Vinson M, Crocker PR, Jones EY (1998) Crystal structure of the N-terminal domain of sialoadhesin in complex with $3^{\prime}$ sialyllactose at 1.85 A resolution. Mol Cell 1: 719-728.

45. Zhuravleva MA, Trandem K, Sun PD (2008) Structural implications of Siglec-5mediated sialoglycan recognition. J Mol Biol 375: 437-447.

46. Attrill H, Imamura A, Sharma RS, Kiso M, Crocker PR, et al. (2006) Siglec-7 undergoes a major conformational change when complexed with the alpha $(2,8)$ disialylganglioside GT1b. J Biol Chem 281: 32774-32783.

47. Chung MC, Wines BD, Baker H, Langley RJ, Baker EN, et al. (2007) The crystal structure of staphylococcal superantigen-like protein 11 in complex with sialyl Lewis $\mathrm{X}$ reveals the mechanism for cell binding and immune inhibition. Mol Microbiol 66: 1342-1355.

48. Baker HM, Basu I, Chung MC, Caradoc-Davies T, Fraser JD, et al. (2007) Crystal structures of the staphylococcal toxin SSL5 in complex with sialyl Lewis $\mathrm{X}$ reveal a conserved binding site that shares common features with viral and bacterial sialic acid binding proteins. J Mol Biol 374: 1298-1308.

49. Imberty A, Varrot A (2008) Microbial recognition of human cell surface glycoconjugates. Curr Opin Struct Biol 18: 567-576.

50. Altschul SF, Gish W, Miller W, Myers EW, Lipman DJ (1990) Basic local alignment search tool. J Mol Biol 215: 403-410.

51. Chenna R, Sugawara H, Koike T, Lopez R, Gibson TJ, et al. (2003) Multiple sequence alignment with the Clustal series of programs. Nucleic Acids Res 31: $3497-3500$

52. De Greve H, Wyns L, Bouckaert J (2007) Combining sites of bacterial fimbriae. Curr Opin Struct Biol 17: 506-512.

53. Sotomayor M, Schulten K (2008) The allosteric role of the $\mathrm{Ca} 2+$ switch in adhesion and elasticity of C-cadherin. Biophys J 94: 4621-4633.

54. Sokurenko EV, Vogel V, Thomas WE (2008) Catch-bond mechanism of forceenhanced adhesion: counterintuitive, elusive, but ... widespread? Cell Host Microbe 4: 314-323.

55. Pyburn TM, Yankovskaya V, Bensing BA, Cecchini G, Sullam PM, et al. (2010) Purification, crystallization and preliminary X-ray diffraction analysis of the carbohydrate-binding region of the Streptococcus gordonii adhesin GspB. Acta Crystallogr F Struct Biol Cryst Commun 66: 1503-1507.

56. Otwinowski Z, Minor W (1997) Processing of X-ray diffraction data collected in oscillation mode. Macromolecular Crystallography, Pt A 276: 307-326.

57. Bailey S (1994) The Ccp4 Suite - Programs for Protein Crystallography. Acta Crystallogr D Biol Crystallogr 50: 760-763.

58. Sheldrick GM (2008) A short history of SHELX. Acta Crystallogr A 64: $112-122$.

59. de La Fortelle E, Bricogne G (1997) Maximum-likelihood heavy-atom parameter refinement for multiple isomorphous replacement and multiwavelength anomalous diffraction methods. Macromolecular Crystallography, Pt A 276: 472-494.

60. Bailey S (1994) The Ccp4 Suite - Programs for Protein Crystallography. Acta Crystallogr D Biol Crystallogr 50: 760-763.

61. Adams PD, Afonine PV, Bunkoczi G, Chen VB, Davis IW, et al. (2010 PHENIX: a comprehensive Python-based system for macromolecular structure solution. Acta Crystallogr D Biol Crystallogr 66: 213-221.

62. Mccoy AJ, Grosse-Kunstleve RW, Adams PD, Winn MD, Storoni LC, et al. (2007) Phaser crystallographic software. J Appl Crystallogr 40: 658-674.

63. Brunger AT (2007) Version 1.2 of the Crystallography and NMR system. Nat Protoc 2: 2728-2733.

64. Emsley P, Cowtan K (2004) Coot: model-building tools for molecular graphics. Acta Crystallogr D Biol Crystallogr 60: 2126-2132.

65. Murshudov GN, Vagin AA, Dodson EJ (1997) Refinement of macromolecular structures by the maximum-likelihood method. A Acta Crystallogr D Biol Crystallogr 53: 240-255. 
66. Potterton E, Briggs P, Turkenburg M, Dodson E (2003) A graphical user interface to the CCP4 program suite. Acta Crystallogr D Biol Crystallogr 59: 1131-1137.

67. Schuttelkopf AW, van Aalten DM (2004) PRODRG: a tool for high-throughput crystallography of protein-ligand complexes. Acta Crystallogr D Biol Crystallogr 60: $1355-1363$.

68. DeLano WL (2002) The PyMOL Molecular Graphics System.

69. Hayward S, Berendsen HJ (1998) Systematic analysis of domain motions in proteins from conformational change: new results on citrate synthase and T4 lysozyme. Proteins 30: 144-154.

70. Schwarz JB, Kuduk SD, Chen XT, Sames D, Glunz PW, et al. (1999) A broadly applicable method for the efficient synthesis of alpha-O-linked glycopeptides and clustered sialic acid residues. J Am Chem Soc 121: 2662-2673.
71. Mitchell J, Siboo IR, Takamatsu D, Chambers HF, Sullam PM (2007) Mechanism of cell surface expression of the Streptococcus mitis platelet binding proteins PblA and PblB. Mol Microbiol 64: 844-857.

72. Takamatsu D, Bensing BA, Sullam PM (2004) Genes in the accessory sec locus of Streptococcus gordonii have three functionally distinct effects on the expression of the platelet-binding protein GspB. Mol Microbiol 52: 189-203.

73. Bensing BA, Takamatsu D, Sullam PM (2005) Determinants of the streptococcal surface glycoprotein GspB that facilitate export by the accessory Sec system. Mol Microbiol 58: 1468-1481.

74. Alphey MS, Attrill H, Crocker PR, van Aalten DM (2003) High resolution crystal structures of Siglec-7. Insights into ligand specificity in the Siglec family. J Biol Chem 278: 3372-3377.

75. Kleywegt GJ (1996) Use of non-crystallographic symmetry in protein structure refinement. Acta Crystallogr D Biol Crystallogr 52: 842-857. 